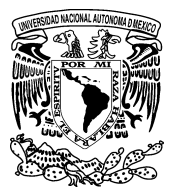

\title{
Inteligencia de negocios: estrategia para el desarrollo de competitividad en empresas de base tecnológica
}

\author{
Business intelligence: Strategy for competitiveness development in \\ technology-based firms
}

Eduardo Ahumada Tello* y Juan Manuel Alberto Perusquia Velasco

Universidad Autónoma de Baja California, México

Recibido el 4 de diciembre de 2013; aceptado el 29 de octubre de 2014

Disponible en Internet el 29 de octubre de 2015

\begin{abstract}
Resumen
La necesidad de realizar una valoración sobre el rol de los activos intangibles dentro de las organizaciones hace que sea necesario establecer estrategias entre la creación de valor con base en el conocimiento y los mecanismos de adquisición de este en las empresas. En esta investigación se plantea la problemática de establecer elementos que desarrollen la capacidad de fortalecer el conocimiento que las empresas adquieren a través de acciones centradas en los sistemas de información, la innovación y el proceso de la toma de decisiones, todo coadyuvando a la ampliación de la inteligencia de negocios (Business Intelligence) como un factor fundamental en la competitividad empresarial. Es una investigación mixta: entrevistas a profundidad en el aspecto cualitativo y cuestionario en el aspecto cuantitativo; en empresas del sector de tecnologías de información y comunicación. Los hallazgos principales son: a) el conocimiento es el activo de mayor valor en las empresas; b) el entorno de negocios es fundamental para la competitividad; c) la innovación, los sistemas de información y los procesos de toma de decisiones son parte de la inteligencia de negocios que
\end{abstract}

\footnotetext{
* Autor para correspondencia.

Correo electrónico: eahumada@uabc.edu.mx (E. Ahumada Tello).
}

La revisión por pares es responsabilidad de la Universidad Nacional Autónoma de México. 
requieren las empresas, y c) la inteligencia de negocios desarrolla la competitividad a partir de la gestión del conocimiento.

Derechos Reservados (C) 2015 Universidad Nacional Autónoma de México, Facultad de Contaduría y Administración. Este es un artículo de acceso abierto distribuido bajo los términos de la Licencia Creative Commons CC BY-NC-ND 4.0.

Palabras clave: Inteligencia de negocios; Competitividad empresarial; Gestión del conocimiento; Innovación de negocios; Procesos de toma de decisiones

\begin{abstract}
The need for an assessment of the role of intangible assets in organizations makes it necessary to establish strategies between value creation based on knowledge and the mechanisms developed for their acquisition in firms. This research raises the question of establishing elements to develop the ability to strengthen business knowledge acquired through actions focused on information systems, innovation and decision making process, all contributing to the expansion of business intelligence as a key factor in business competitiveness. It is a mixed research: by using in-depth interviews in qualitative approach and a questionnaire in the quantitative approach, in firms from the Information and Communication Technologies (ICT) Economical Sector. The main findings are: (i) knowledge is the most valuable asset in modern ICT firms; (ii) business environment is essential for competitiveness; (iii) innovation, information systems and decision making process are part of business intelligence and is required by firms, and (iv) business intelligence develops competitiveness from knowledge management point of view.

All Rights Reserved () 2015 Universidad Nacional Autónoma de México, Facultad de Contaduría y Administración. This is an open access item distributed under the Creative Commons CC License BY-NC-ND 4.0.

Keywords: Business intelligence; Business competitiveness; Knowledge management; Business innovation; Decision making process
\end{abstract}

\title{
Antecedentes
}

El conocimiento surge cuando un ente lleva a cabo la percepción de que su propia experiencia y capacidad le ha dado la posibilidad de interpretar información que esté recibiendo en un momento determinado (Zapata-Cantú, 2004). El conocimiento deriva de información, así como la información deriva de los datos. Existe una relación directa entre datos, información y conocimiento. Si la información se transforma en conocimiento, entonces ha ocurrido la intervención de un ente inteligente. Las acciones de generación del conocimiento se producen en los seres humanos (Davenport y Prusak, 2001). Las tecnologías de información y comunicación (TIC), a pesar de su capacidad de procesamiento de datos y creación de información, no pueden crear el conocimiento; este solo es posible a partir de la intervención de un ser humano (Bueno, 2000).

El conocimiento se presenta como una alternativa para el desarrollo social, el que surge a partir de las experiencias empíricas pero que posteriormente se puede llevar a su acumulación y dar origen a nuevas formas de entender algún fenómeno conocido, y da lugar a la consecución de logros que pueden, en determinado momento mejorar la calidad de vida de los hombres que conforman una sociedad determinada (Shapiro y Varian, 1999).

En la figura 1 se ilustra la forma en que, partiendo de ciertas premisas que son sujetas tanto a la creencia como a las verdades que se consideran ciertas, se logra llegar a la creación de «conocimiento». Este, definitivamente, está sujeto al contexto que lo condiciona a ser parte de 


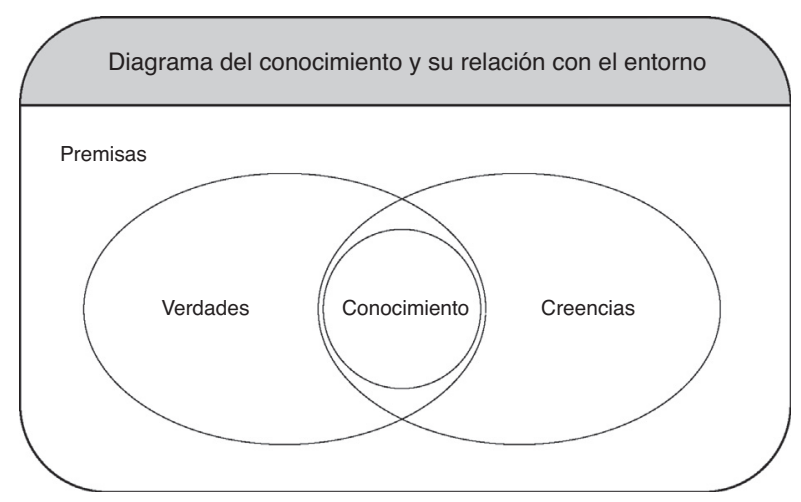

Figura 1. Conocimiento basado en la interacción como premisas filosóficas.

Fuente: elaboración propia.

lo que se manifiesta como posible. Cada autor, investigador, «observador» o sociedad tienen en común antecedentes históricos, filosóficos y cosmogónicos que definen todo lo creíble y lo considerado verdadero (Michel, 2006).

Michel (2006) escribe sobre la variabilidad de lo conocido. La visión cosmogónica del conocimiento varía de acuerdo al observador y su propia percepción de la realidad. Esta no es correcta ni incorrecta, simplemente es. Esto lo expresa Michel cuando cita a Pérez Tamayo:

«En un sentido muy real, el científico no descubre la realidad, sino que la inventa, y por medio de observaciones y experimentos — que también contiene fuertes elementos subjetivosestablece y perfecciona un grupo de modelos o teorías posibles...»

El pensamiento de Pérez Tamayo refuerza la idea del subjetivismo que rodea a toda expresión de saber. Nos es dado creer lo que otros dicen que debemos creer (Goebbels, 1938). El grupo social crea bajo los parámetros de las creencias y verdades aceptadas un entorno donde se insertan los descubrimientos, premisas e ideas que son empáticas, y por ende la realidad que se percibe determina el conocimiento que se genera, uno se desprende del otro y así se forman las relaciones en ambas direcciones: por un lado la realidad aceptada y por el otro los descubrimientos o inventos que forjan el conocimiento que refuerza dicha realidad.

En las organizaciones se presenta una situación que versa en el manejo de la información, que cada vez es más numerosa y difícil de categorizar. La competitividad de las empresas considera importante que estas aprendan y que con el tiempo puedan replicar el conocimiento que se concentra en ellas a partir de los diferentes agentes involucrados en su operación, pudiendo ser empleados, directivos, clientes, etc. (Ahumada-Tello, Zárate Cornejo, Plascencia López y Perusquia-Velasco, 2012). Es por ello que en esta investigación se aborda la problemática de lograr que las empresas sean inteligentes, aquellas que mediante el uso de estrategias basadas en el conocimiento desarrollan acciones de inteligencia de negocios, o, en otras palabras, que sean capaces de aprender a partir de sus miembros y sostener este conocimiento para la generación y transferencia valor (Berg de Valdivia, 2007).

Los elementos en que se sustenta la conceptualización de inteligencia de negocios son los sistemas de información (Elbashir, Collier y Sutton, 2011; Deng y Chi, 2012; Bara et al., 2009), los mecanismos de innovación (Nemutanzhela e Iyamu, 2011; Railean, 2011; Kumar y Puranam, 2012) y los procesos de toma de decisiones (Chaabouni y Triki, 2013; Ángel, 2010). En cada uno 

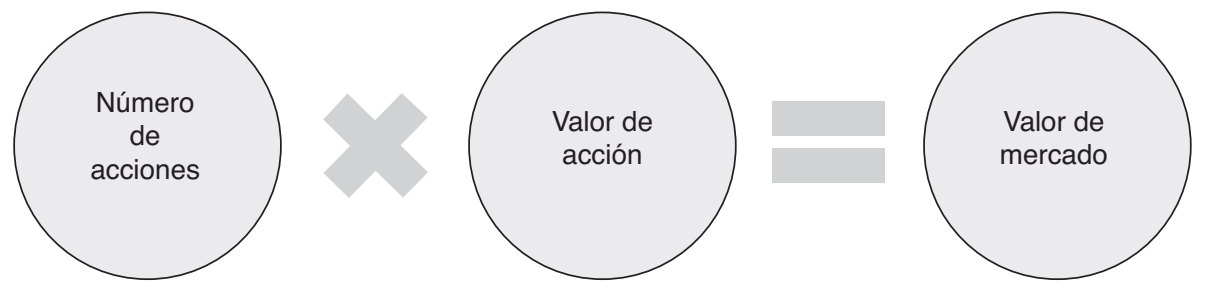

Figura 2. Valor tradicional de mercado de las empresas.

Fuente: Pavez Salazar, 2000.

de ellos se implementan estrategias que pueden llevar a la organización a adquirir conocimiento y a mejorar la manera en que este incrementa el valor de los productos y servicios que se ofrecen, en este caso las empresas de base tecnológica en Tijuana, B.C.

\section{Marco de referencia}

\section{El conocimiento y las organizaciones}

Para entender la posición actual del conocimiento hacia el interior de las empresas es importante considerar las estructuras del valor de las organizaciones. Antes de definir el valor de una organización se debe entender el significado de este concepto. Desde un punto de vista económico, tal como el de los accionistas, se define como el valor monetario de las acciones de la empresa. Esta definición se puede visualizar en la figura 2.

Es posible que se pueda llegar a considerar que el valor de mercado de una empresa se encuentre fuera del valor real o contable de la misma, debido a la volatilidad de los precios de las acciones, pero es interesante y propenso a investigación cuando el valor de las empresas se sitúa muy por encima de su valor contable. Sin embargo, en los nuevos paradigmas de negocios que han surgido a través del uso de las tecnologías de información y comunicación como centro de las actividades generadoras de utilidades, las empresas empiezan a perfilar una necesidad estratégica el atender y construir sistemas de evaluación que les permitan asignar un valor a las actividades sustentadas en el conocimiento (Sallis y Jones, 2002).

\section{El valor del conocimiento en las organizaciones}

Siguiendo la perspectiva del valor empresarial del conocimiento, se presenta así mismo la necesidad de determinar qué impacto tiene este en el desarrollo de la competitividad (Duran, 2002). En el ámbito organizacional surge esta capacidad cuando una organización es capaz de producir bienes y servicios de calidad sin denotar una distinción entre los que sean de tipo tangible o intangible. Este enfoque indica la capacidad de producir valor; el conocimiento y su gestión, por lo tanto, se convierten en uno de los factores clave para la innovación y la consolidación de una organización en el entorno global de los negocios (Araya Guzmán, 2004).

Toda organización busca el continuo mejoramiento de sus relaciones con sus clientes, empleados, accionistas y demás stakeholders ${ }^{1}$ involucrados con la organización. Los beneficios pueden

\footnotetext{
1 Término anglosajón comúnmente utilizado para señalar las partes interesadas en el desempeño organizacional. Pueden ser los accionistas, los empleados, los proveedores, las autoridades gubernamentales o los organismos indirectos, entre otros.
} 


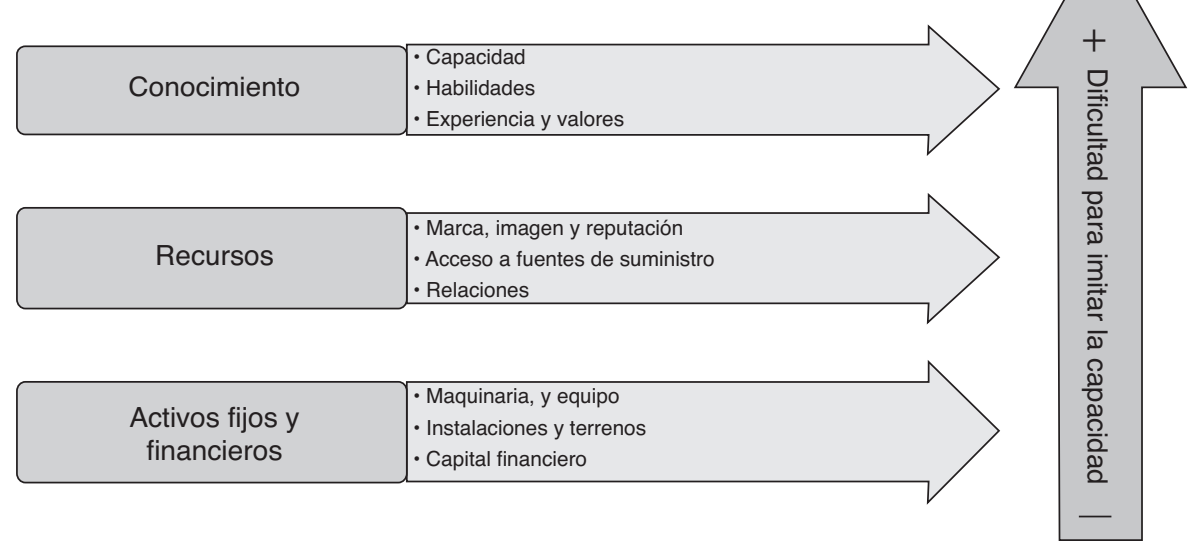

Figura 3. Valor del conocimiento.

Fuente: Elaboración propia a partir de Del Brío, Fernández y Junquera (2005)

ser sociales o económicos, el reto se perfila en la necesidad de lograr una mayor eficiencia y eficacia en sus procesos de producción, principalmente si estos se sustentan en los activos intangibles que son generados a partir de una estrategia de desarrollo basada en el conocimiento. En la figura 3 se ilustra el enfoque de la conformación de valor hacia el conocimiento.

El conocimiento es la estrategia con mayor dificultad de imitación. Aquellas tácticas que se sustenten en replicar los elementos respaldados en estrategias sobre activos fijos y financieros - tales como maquinaria y equipo, instalaciones y terrenos, así como capital financiero-, o de la misma manera las que busquen generar recursos similares a la competencia - como por ejemplo la generación de marca, imagen y reputación, acceso a fuentes de suministro y las relaciones comerciales-, son relativamente más sencillas de replicar y de esta manera eliminar la ventaja competitiva (Tiwana, 2002).

Sin embargo, las acciones que se perfilan como conocimiento de la empresa - o en este caso como inteligencia de negocios - se dividen en: experiencia y valores, habilidades y capacidades. Se convierten en características difícilmente igualables que en gran medida otorgan ventajas competitivas a las empresas u organizaciones que buscan fortalecer estas áreas de oportunidad.

Para reforzar el concepto de valor en el conocimiento se puede mencionar a Nonaka y Takeuchi (1999), autores que enfatizan la importancia que tiene en las empresas modernas la creación de conocimiento. En este caso, los autores del factor de conocimiento hacen énfasis en que: «[...] no se busca la creación conocimiento sino [. . .] se pretende añadir valor».

A partir del valor que el conocimiento brinda a la organización, surge la teoría basada en el conocimiento (knowledge based view), que considera a la empresa como una comunidad social representante de un cúmulo de información, experta en la creación, la transmisión interna y su aplicación para generar conocimiento $y$, por consiguiente, ventaja en el proceso de competencia de mercado (Berg de Valdivia, 2007).

Así mismo, se establece que la principal fuente de ventaja competitiva duradera de la empresa reside en el conjunto de información, que al ser analizada facilita cierto conocimiento. Y cuando estas empresas son capaces de crear, aplicar y replicar constantemente estos conocimientos 
específicos, se establecen los lineamientos organizacionales que permiten alcanzar objetivos estratégicos en su planteamiento competitivo (Chesbrough, 2011).

\section{Gestión del conocimiento}

Es en este nuevo ámbito de la economía del conocimiento que se promueve el valor de los intangibles como centro de las acciones y estrategias empresariales; la gestión del conocimiento se ha convertido en uno de los principales temas de investigación y en el paradigma de gestión por excelencia en el campo de la organización y gestión de instituciones empresariales (Gallego y Ongallo, 2004). Los siguientes autores remarcan la importancia de la gestión del conocimiento:

«Todas las organizaciones saludables generan y usan conocimiento. A medida que las organizaciones interactúan con sus entornos, absorben información, la convierten en conocimiento y llevan a cabo acciones sobre la base de la combinación de ese conocimiento y de sus experiencias, valores y normas internas. Sienten y responden. Sin conocimiento, una organización no se podría organizar a sí misma [...]» (Davenport y Prusak, 2001).

«[.. . ] la capacidad de una compañía para generar nuevos conocimientos, diseminarlos entre los miembros de la organización y materializarlos en productos, servicios y sistemas. La creación de conocimiento organizacional es la clave del proceso peculiar a través del cual estas firmas innovan. Son especialmente aptas para innovar continuamente, en cantidades cada vez mayores y en espiral [generando ventaja competitiva para la organización]» (Nonaka y Takeuchi, 1999).

La aparición y la creciente importancia del conocimiento como un factor de producción hacen que el desarrollo de tecnologías, de metodologías, de innovación y de estrategias para su medición, creación y difusión se convierta en prioridad en las organizaciones en la nueva denominación de sociedad del conocimiento (Michelo et al., 2008). Sin embargo, también se puede considerar que ha sido precisamente el desarrollo de estas el que ha convertido el conocimiento en un elemento indispensable para el desarrollo económico y social (Bueno, 2008).

\section{Inteligencia de negocios}

A partir de la gestión del conocimiento, surge el concepto de inteligencia de negocios (Business Intelligence, inteligencia empresarial o inteligencia de negocios); se llama así al conjunto de estrategias, acciones y herramientas enfocadas a la administración y creación de conocimiento mediante el análisis de datos existentes en una organización o empresa (Ahumada-Tello et al., 2012).

Es precisamente en la gestión del conocimiento donde se sustentan estas estrategias que permiten seguir un conjunto de acciones que la empresa inteligente puede emprender, y que le conceden una ventaja sobre sus competidores, principalmente porque el valor agregado a los servicios o productos que son consecuencia de estas acciones desarrollan una eficiencia en su producción y una eficacia en su funcionamiento que difícilmente pueden ser replicadas por aquellas que no tienen estos procesos o estrategias definidas (Larson, 2009).

En este documento se definen específicamente como acciones de inteligencia de negocios las operaciones que se involucran con los sistemas de información (Deng y Chi, 2012; Elbashir et al., 2011), con los procesos de innovación (Medellín Cabrera, 2010; Chesbrough, 2011) y con el desarrollo de procedimientos para generar un proceso de toma de decisiones inteligente. Los 
sistemas de información se definen como los elementos electrónicos del manejo de la información; la influencia de ellos sobre los procesos de negocio y sus implicaciones prácticas para la generación de conocimiento. De esta manera, son los diferentes sistemas que se estén utilizando para llevar adelante la operación de la empresa.

La innovación se define como las acciones u operaciones que promueven la mejora de cualquiera de los productos o servicios que produce la empresa (Chesbrough, 2011), pudiendo ser estos de tipo tecnológico, operacional, administrativo o de estrategia empresarial (Chesbrough, 2010). Así mismo son las políticas de promoción que la empresa tenga para llevar a cabo valoración de procesos, de productos, de personas en la organización y de acumulación o concentración de información en sus procesos productivos.

Finalmente, los procesos de toma de decisión se definen como la forma en que la gerencia llega a determinar las acciones a seguir, si estos son elaborados en base a una metodología preestablecida o si existen lineamientos que apoyen la evaluación de los resultados de las mismas (Chaabouni y Triki, 2013). Son los mecanismos, documentos, procesos y políticas que promueven la toma de decisiones inteligentes en la organización (Moss y Atre, 2003).

\section{Modelos de gestión empresarial}

Ahora bien, para lograr establecer metodologías y modelos de gestión que se orienten al desarrollo de estrategias de inteligencia de negocios es necesario analizar los modelos que tienen mayor difusión y que a la vez reconozcan el conocimiento como elemento primordial en el desarrollo de las organizaciones.

Primeramente, un modelo se define como la representación de un fenómeno desde la perspectiva del observador. Este surge al analizar con perspectiva sistémica el problema. Los modelos son diseñados para describir, comprender, explicar y predecir el comportamiento de las partes que componen el fenómeno o sus componentes (Mircea, 2009).

En este sentido, los modelos de gestión empresarial son relevantes debido a la importancia del enfoque que se aplique a la gestión del conocimiento y del valor intangible de las organizaciones. A continuación se mencionan varios de los modelos de mayor difusión en los negocios.

\section{Modelo Fundación Europea para la Administración de Calidad}

El modelo Fundación Europea para la Administración de Calidad (EFQM) es uno de los modelos empresariales que se utilizan en Europa de forma común. En la versión Excelencia 2000 (EFQM, 2010) hace mención de la importancia del conocimiento, la innovación y los procesos de aprendizaje para llegar a la excelencia empresarial. Este modelo tiene como objetivo ofrecer a las empresas una metodología que las lleve al mejoramiento de sus estrategias para el logro de resultados organizacionales (fig. 4).

En el modelo EFQM se encuentra señalada en ambos sentidos la importancia de las personas en los procesos de generación de conocimiento, lo que establece una relación con la inteligencia de negocios, puesto que es allí donde la organización mantiene de manera permanente los resultados del modelo. Es desde la perspectiva de la innovación y de los resultados de este proceso que los agentes facilitadores pueden mejorar los resultados, y de esta manera se infiere que la empresa también aprende durante estas fases de adquisición de valor. El liderazgo, orientado hacia los resultados, pero considerando de importancia equilibrada a las personas, a la política y a la estrategia organizacional, a las alianzas y los recursos, a los procesos internos, a los clientes y 


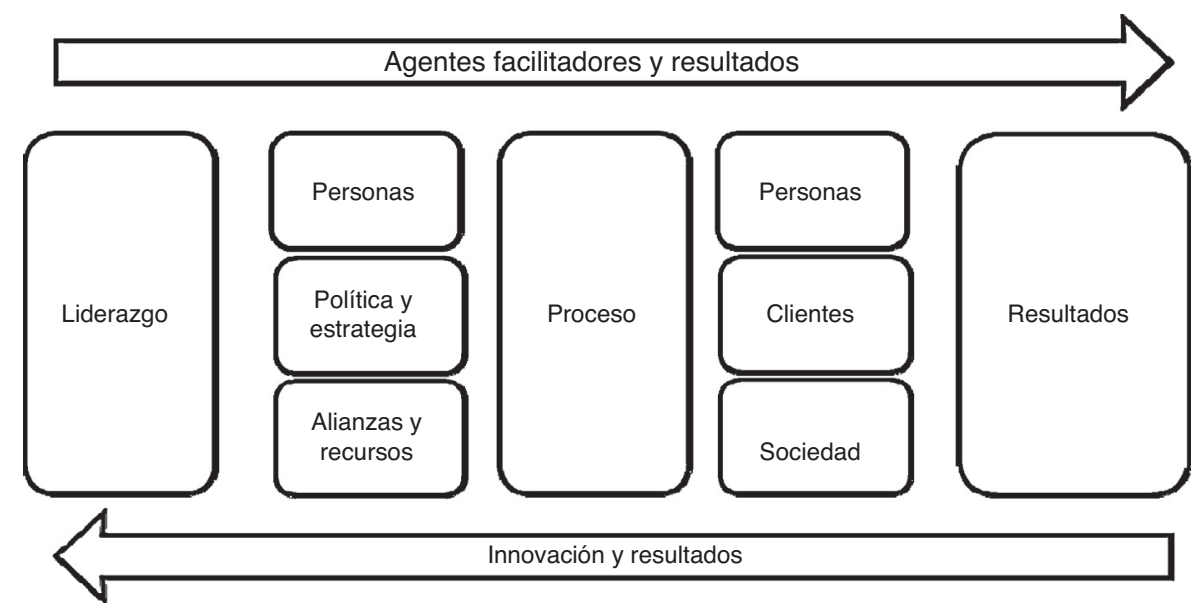

Fuente: EFQM, 2010.

Figura 4. Modelo EFQM.

al impacto en la sociedad, pretende mejorar el sistema de producción elevando la calidad de los productos y servicios bajo la influencia de una perspectiva de gestión de conocimiento.

\section{Modelo de Cuadro de Mando Integral}

Este modelo, propuesto por Kaplan y Norton (2006), establece una serie de indicadores capaces de ofrecer una visión global de la empresa, de activos tanto tangibles como intangibles, por medio de los indicadores financieros del modelo. Este modelo incluye la posibilidad de gestionar valores intangibles, tales como el conocimiento. Sus funciones son:

- Clarificar la visión y la estrategia organizacional.

- Comunicar los objetivos estratégicos y aumentar la comunicación organizacional.

- Alinear las iniciativas estratégicas.

- Aumentar la comunicación organizacional.

- Facilitar la toma de decisiones.

Se muestra como una de sus vertientes principales el apoyo a la toma de decisiones, y se mencionan 4 indicadores que aparecen en la figura 5. La visión estratégica se sustenta en las diferentes visiones o perspectivas: la de los clientes, la financiera, la interna y la de aprendizaje continuo. Esta última se contempla como la adecuación de la inteligencia de negocios en los procesos que generan la visión organizacional; de esta manera, los modelos de gestión como este refuerzan el uso del conocimiento en la generación de estrategia.

\section{Modelo Intelecto}

Responde al interés de medir el capital intelectual en las organizaciones. Este pretende ofrecer información relevante para la toma de decisiones y facilitar información a terceros sobre el valor de la empresa. Se pretende también acercar el valor implícito de la empresa a su valor de mercado, así como informar sobre la capacidad de la organización para generar resultados sostenibles, mejoras continuas y crecimiento a largo plazo (fig. 6). 


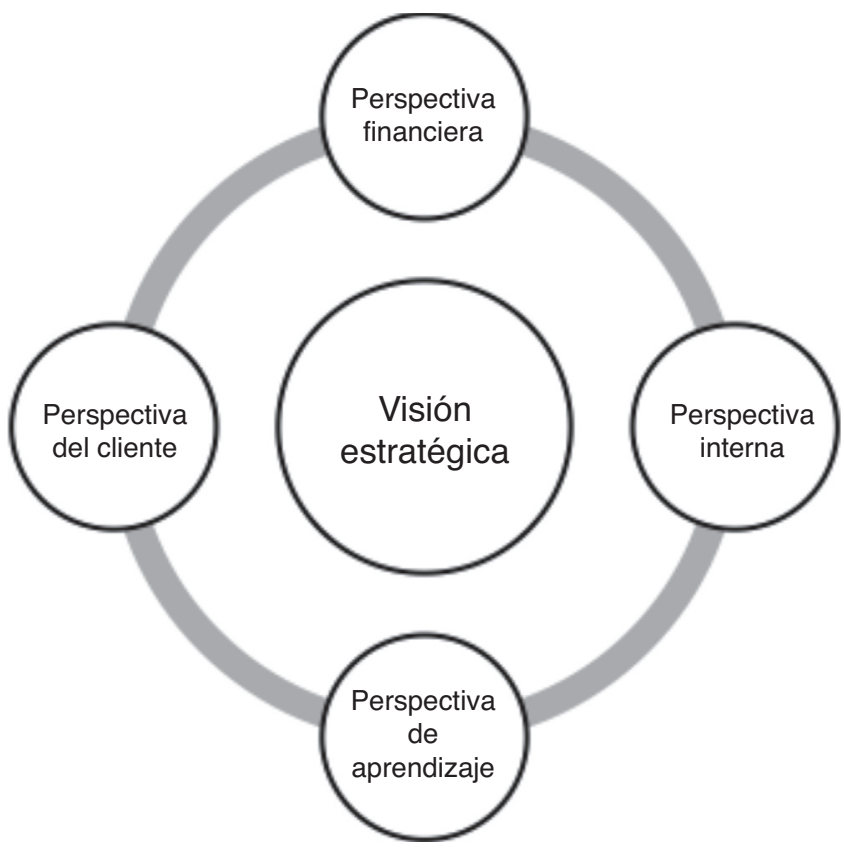

Fuente: Kaplan y Norton, 2006.

Figura 5. Cuadro de mando integral.

Este modelo antecede a la visión de una inteligencia de negocios. Los elementos del capital intelectual que menciona — capital humano, capital relacional y capital estructural — forman parte de los factores supeditados a la adquisición de conocimiento en la organización. Se plantea en consecuencia que en el futuro estratégico la organización eleva los índices de cada factor de manera que la organización mantenga sus ventajas competitivas con apoyo del capital intelectual.

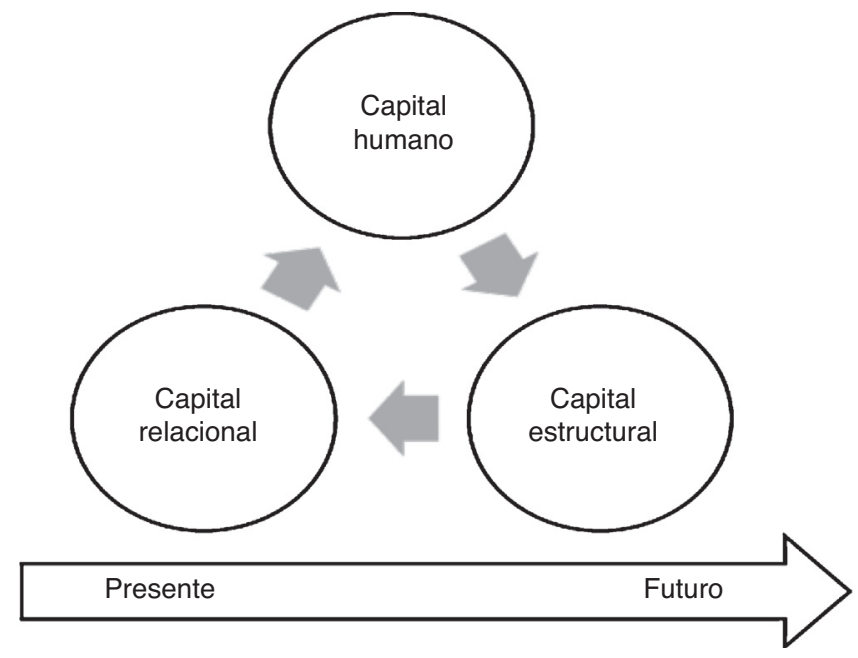

Fuente: Kaplan y Norton, 2006.

Figura 6. Bloques de capital intelectual. 


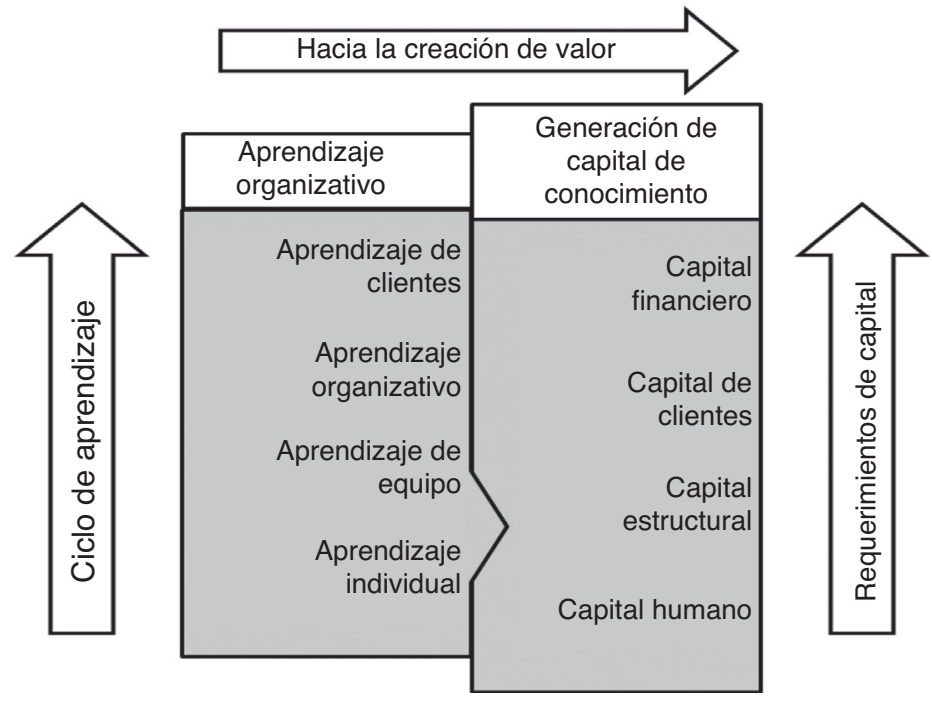

Fuente: Saint-Onge, 1996.

Figura 7. Modelo Saint-Onge.

\section{Modelo Saint-Onge}

Hubert Saint-Onge expresa que el conocimiento es «como la energía eléctrica que fluye entre los activos intangibles de la empresa para alimentar el capital humano, el capital estructural y el capital cliente» (Saint-Onge, 1996). Estos son los elementos que conforman los activos intangibles de la empresa:

- El capital humano, constituido por los conocimientos, experiencias, etc., de los individuos de la organización.

- El capital estructural, integrado por la estructura organizativa, los procesos, los equipos, programas, bases de datos y todo lo que forma parte de la capacidad organizacional de una empresa.

- El capital cliente, representado por las relaciones que se desarrollan con los clientes claves de la organización.

El modelo Saint-Onge se centra en el conocimiento tácito de la empresa y en cómo renovarlo y gestionarlo de la manera más eficaz. Se fundamenta en la idea de que al comprender el conocimiento tácito, la empresa puede encontrar formas de generar una cohesión interna dinámica que mejore el rendimiento futuro de la organización. El creador de este modelo incluye el concepto de capital cliente, por considerarlo un factor determinante. En la figura 7 se ilustra el modelo.

\section{Modelo Skandia}

Este modelo parte de la idea de que el valor de la empresa no se refleja solo contando los activos tangibles; bajo este argumento es que se agrega el factor financiero al modelo. Además de los indicadores tradicionales enfocados en los valores tangibles, en este modelo se incluye la evaluación de rendimiento, rapidez y calidad (Edvinsson, Hofman-Bang y Jacobsen, 2005). 


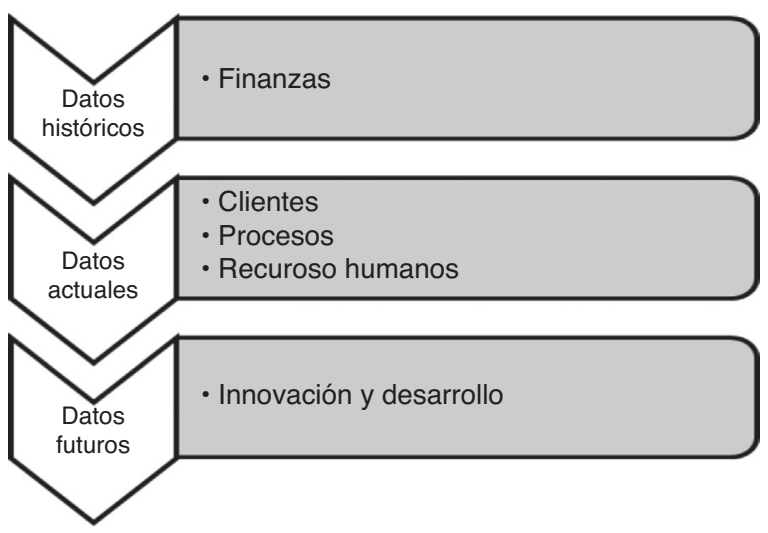

Fuente: Saint-Onge, 1996.

Figura 8. Modelo Skandia.

Tabla 1

Factores de fracaso para un modelo de gestión del conocimiento

\begin{tabular}{ll}
\hline \multicolumn{2}{c}{ Factores clave del fracaso de la gestión del conocimiento } \\
\hline Ausencia de objetivos & Falta de planificación \\
Falta de cultura adecuada & Confusión conceptual \\
Responsabilidad difusa & Contextualización \\
\hline
\end{tabular}

Fuente: Davenport y Prusak, 2001; Rodríguez Gómez, 2006.

El modelo incluye el análisis de las finanzas como parte de los resultados de acciones pasadas tomadas en la empresa en relación a la creación de valor; los clientes, los recursos humanos y los procesos como parte de los datos actuales, para finalizar con las acciones de renovación y desarrollo como efectos en el futuro de la empresa. Estas secciones se indican en la figura 8.

\section{Factores clave del éxito de modelo de gestión del conocimiento}

Aunque los factores que, en función del contexto particular, pueden determinar el éxito de un proceso de gestión del conocimiento son muchos y variados, en la tabla 1 se enumeran los definidos para lograr el éxito de un proyecto de gestión del conocimiento.

Tabla 2

Factores de éxito para un modelo de gestión del conocimiento

Factores clave del éxito de la gestión del conocimiento

\begin{tabular}{ll}
\hline Cultura orientada al conocimiento & Claridad de objetivo y lenguaje \\
Infraestructura técnica e institucional & Prácticas de motivación \\
Respaldo del personal directivo & Estructura de conocimiento \\
Vínculo con el valor económico o valor de mercado & Múltiples canales para la transferencia de \\
Orientación del proceso & conocimiento \\
\hline
\end{tabular}

Fuente: Davenport y Prusak, 2001; Rodríguez Gómez, 2006. 
Factores clave del fracaso de modelo de gestión del conocimiento

Aunque los factores que, en función del contexto particular, pueden determinar el éxito de un proceso de gestión del conocimiento son muchos y variados, en la tabla 2 se enumeran los definidos que provocan el fracaso de un proyecto de gestión del conocimiento.

\section{Inteligencia de negocios y gestión del conocimiento}

Desde que Freire (1973) elaborara sus teorías sobre la razón de ser del conocimiento y cómo este otorga libertad al individuo, se ha recorrido un largo camino hacia el reconocimiento de este como un valor activo de las universidades, empresas, gobiernos o cualquier otra organización que lo posea (De la Fuente, 2002). La relevancia de la creación y transferencia del conocimiento hace importante que este sea gestionado de una forma eficiente y que llegue a todos los actores que lo requieran dentro de una sociedad (UNESCO, 2005). Ahora, al conocimiento se le considera como un alto valor competitivo en las organizaciones, y su eficiente gestión, un valor agregado de la misma (Zittoun, 2008; Kogut y Zander, 1992; Villarreal, 2006).

En la década de los noventa ya se establecía la importancia de la gestión en el desarrollo de las empresas (Hansen, Nohria, y Tierney, 1999). Y fue Grant (1996) quien afirmó que las organizaciones basadas en el conocimiento emergen y trascienden en el entorno académico y empresarial.

Los estudios para tratar de comprender la composición y la importancia del conocimiento, así como la relevancia de su almacenamiento, transformación y distribución, han incluido el análisis social como un marco que permite entender los elementos individuales que componen el conocimiento (Navarro y Bonilla, 2003). La etnografía analiza principalemente, mediante la observación de las características concernientes a una cultura en especial, las formas de interrelacionarse en esos núcleos que generan conocimiento, y por consiguiente ayuda a comprender su creación (Kane, Ragsdell, y Oppenheim, 2006).

Procedimientos y métodos computacionales también forman parte de los factores de apoyo para otorgar valor al conocimiento (Goitia, Sáenz-de-Lacuesta y Bilbao, 2008). La perspectiva de la inteligencia de negocios se sustenta en la organización que aprende, que adquiere el valor de las habilidades y conocimientos particulares de los individuos (capital humano), también de las estructuras organizacionales y condiciones de mercado (capital estructural) y, finalmente, de los procesos de formación de estrategias de vinculación, alianzas y colaboraciones (capital relacional).

Es en este marco referencial que se puede inferir que la inteligencia de negocios depende de la dirección organizacional y de los estilos de liderazgo, para efectuar la implementación adecuada de los sistemas de información, de los mecanismos de innovación y de los procesos para la toma de decisiones que en conjunto se administran por medio de un sistema de gestión del conocimiento. Sin embargo, es a partir de la base de trabajadores, de los procesos y relaciones que se forman entre ellos, así como de la cultura organizacional, que surge la inteligencia de negocios adecuada al modelo de gestión (Medellín Cabrera, 2010).

La figura 9 resume la estructura teórica en este documento. El conocimiento es la base sobre la que se crea un valor; este valor eventualmente requiere ser gestionado, razón por la que se desarrollan modelos de gestión empresarial de manera diversa, adecuándose a cada entorno. Finalmente, surge la noción de inteligencia sobre la base del conocimiento adquirido en las fases previas. Esta ilustración señala una aproximación al concepto de estudio y establece los lineamientos que se siguen para determinar el grado de avance en el concepto de inteligencia de negocios. 


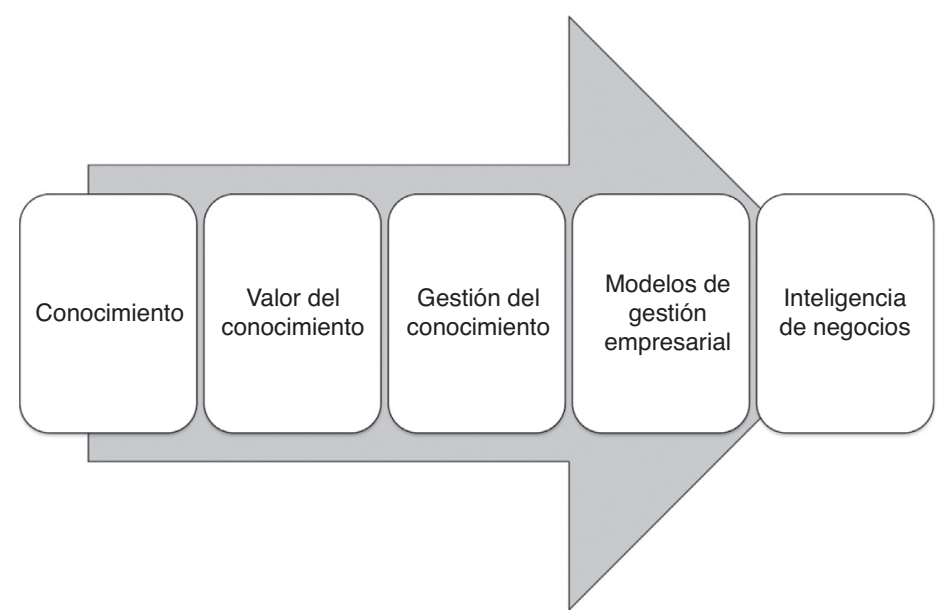

Figura 9. Secuencia teórica sobre inteligencia de negocios.

Fuente: elaboración propia.

\section{Competitividad sistémica}

En sus estudios por parte del Instituto Alemán para el Desarrollo, Esser, Hillebrand, Messner y Meyer-Stamer (1996) definen que la industria se debe sujetar al análisis de su competitividad desde 4 niveles económicos y sociales que se sustentan en un entorno social y, por consiguiente, sistémico.

- Nivel micro: de las empresas y las redes que se forman entre ellas para mejorar sus procesos productivos.

- Nivel meso: del Estado y los actores sociales, mediante el desarrollo de políticas de apoyo y colaboración para fomentar la formación de estructuras y procesos de aprendizaje en la sociedad.

- Nivel macro: sobre las exigencias del entorno que circunda a las empresas y les impone niveles de exigencia en el desempeño y productividad.

- Nivel meta: se refiere a la estructura de patrones organizacionales en el ámbito jurídico, político, económico y de responsabilidad social con miras a la integración estratégica.

Variables de estudio en los niveles de competitividad sistémica

Nivel meta. En este nivel se encuentran los procesos de toma de decisiones en la empresa.

Nivel macro. En este nivel se encuentran los sistemas de información.

Nivel meso. En este nivel se encuentra la innovación.

Nivel micro. En este nivel se encuentran las variables innovación y sistemas de información.

\section{Resumen teórico del trabajo de investigación}

En la tabla 3 se ilustran algunos de los principales autores que sustentan la estrategia de investigación en este documento. 
Tabla 3

Relación de autores y aproximación teórica

\begin{tabular}{ll}
\hline Variable de estudio & Autores principales \\
\hline Inteligencia de negocios & Surma (2011), Larson (2009), Bueno (2008), Moss y Atre (2003) \\
Sistemas de información & Deng y Chi (2012), Elbashir et al. (2011), Bara et al. (2009) \\
Innovación & Medellín Cabrera (2010), Nemutanzhela e Iyamu (2011), Railean (2011), Kumar y \\
& Puranam (2012), Chesbrough (2010, 2011), Carrillo et al. (2012) \\
Toma de decisiones & Chaabouni y Triki (2013), Ángel (2010) \\
Competitividad sistémica & Zittoun (2008), Meyer-Stamer (2008), Villarreal (2006), Esser et al. (1996) \\
\hline
\end{tabular}

Fuente: elaboración propia.

\section{Metodología}

\section{Planteamiento del problema}

En esta investigación se estudia el sector de tecnologías de información en el municipio de Tijuana, B.C., desde la perspectiva dinámica del sector. Así mismo, se analizan los procesos de inteligencia de negocios que forman parte de la gestión del conocimiento como herramienta para determinar su influencia en el desempeño de las empresas que forman parte del sector (Ahumada-Tello, 2011).

Este modelo de evaluación de la competitividad se enfoca en 4 aspectos estudiados por el Instituto Alemán para el Desarrollo: el nivel micro, el nivel macro, el nivel meso y el nivel meta. Cada uno de ellos define aspectos de la empresa-industria analizando desde la cultura de la región hasta los entornos macroeconómicos y las organizaciones de cooperación que se forman (MeyerStamer, 2008). Estos ofrecen una perspectiva de análisis que es especialmente útil para el estudio de los procesos específicos de interacción local-global que implica el desarrollo regional (García, 2009).

En este sentido cabe destacar que el Instituto Alemán para el Desarrollo se ha encargado de llevar a cabo estudios sobre la «transformación orientada a la sustentabilidad en países en vías de desarrollo», incluyendo a Iberoamérica, centrándose en cómo la transformación orientada a la sostenibilidad se puede conceptualizar y medir, cuáles son sus implicaciones financieras y tecnológicas dentro de la empresa, así como qué factores promueven o dificultan tal transformación, dando énfasis en cómo los países en desarrollo pueden encontrar estrategias que armonicen los objetivos económicos y sociales. De ahí que dichos elementos propuestos por el Instituto (nivel micro, nivel macro, nivel meso y nivel meta) sean utilizados como aspectos a incluir en este modelo.

Ahora bien, la problemática de investigación versa en el uso de la gestión del conocimiento como parte esencial de la ventaja competitiva empresarial (Davenport y Prusak, 2001); es el enfoque de análisis sobre la realidad de que entre mayor conocimiento y mejores prácticas para obtenerlo y administrarlo, mejor será la productividad y, por consecuencia lógica, un aumento de la competitividad en el sector de las tecnologías de información desde el enfoque sistémico (Esser et al., 1996).

La investigación busca responder a la interrogante acerca de los procesos de inteligencia de negocios (Business Intelligence) como factores de la gestión del conocimiento que influyen en la competitividad de las empresas que pertenecen al sector de tecnologías de información en Tijuana, B.C. 


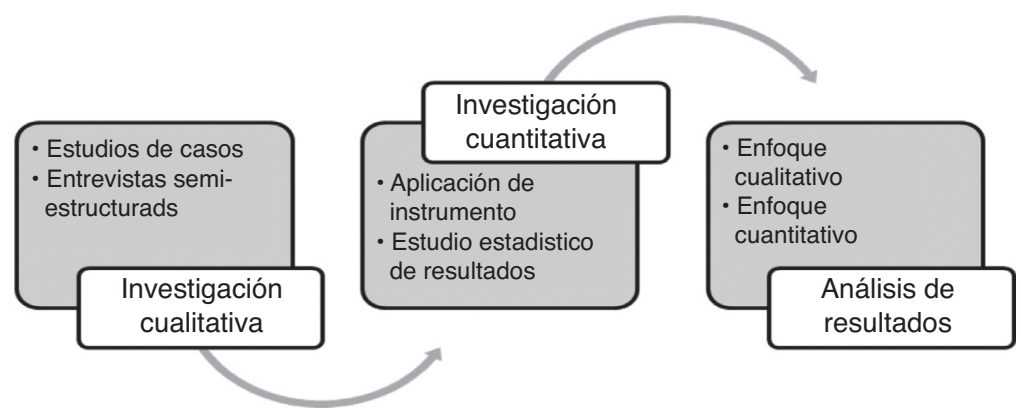

Fuente: elaboración propia.

Figura 10. Proceso de investigación.

\section{Objetivos del estudio}

El objetivo del estudio hace énfasis en describir, analizar y correlacionar los sistemas de información, las acciones promotoras de la innovación y los procesos de toma de decisiones como factores de incremento de la inteligencia de negocios en el desarrollo de competitividad desde el enfoque de la gestión del conocimiento en las empresas del sector de tecnologías de información en Tijuana, B.C.

La investigación se realiza sobre un sector estratégico en la economía nacional (Delgado y Garibotti, 2005), y la importancia radica en la factibilidad que tiene este sector para ayudar al crecimiento de regiones e inclusive naciones mediante la promoción de la tecnología como parte de los lineamientos de una política de desarrollo (Carrillo, Hualde y Villavicencio, 2012). El estudio de las empresas del sector de tecnologías de información desde perspectivas basadas en su valor intangible se sustenta en la gestión del conocimiento (Marsal y Molina, 2002). Para efectos de obtener resultados válidos, se ha recurrido a una estrategia de investigación mixta, esto es, se utilizan técnicas cualitativas y cuantitativas para encontrar resultados congruentes al proceso indagatorio (fig. 10).

\section{Muestra cualitativa}

Durante el proceso cualitativo se llevaron a cabo entrevistas a profundidad en empresas del sector. Este tipo de entrevistas cualitativas en profundidad son no directivas, no estructuradas, no estandarizadas y abiertas; aquí lo que importa es la opinión del entrevistado, su modo de ver el mundo y, por ende, la subjetividad que el individuo imprime en el conocimiento que nos está compartiendo. El nombre, la duración de trabajo y la descripción del proceso de entrevistas realizadas a las organizaciones participantes en este estudio dependieron del acceso y de la pertinencia que tienen cada una de ellas, tomando un total de 14 empresas de base tecnológica.

Cada entrevista tuvo un investigador como entrevistador, y la duración osciló entre los 50 y los $120 \mathrm{~min}$. Las conversaciones fueron audiograbadas y, junto con anotaciones (realizadas durante el evento), conforman la información recabada. La fase cualitativa se expone en la figura 11.

\section{Muestra cuantitativa}

En cuanto al universo objeto de estudio, y tras analizar las características del sector de tecnologías de información en la ciudad de Tijuana, B.C., se decide que formen parte del análisis todas 


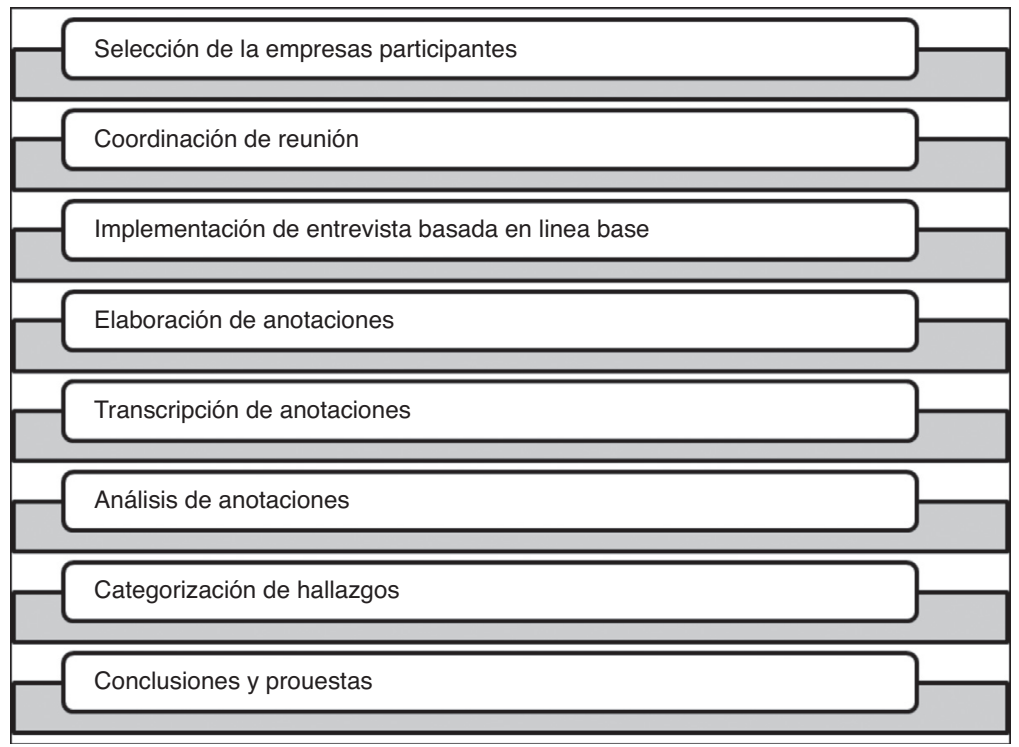

Figura 11. Proceso y metodología de entrevistas no estructuradas.

Fuente: Krueger y Casey, 2000.

las empresas reportadas por el estudio realizado por PRODUCEN en 2007 y 2008 (PRODUCEN, 2008), así que se buscan datos de las empresas en el Clúster de Tecnologías de Información de Baja California, A.C. (ITBaja) y también en la Cámara Nacional de la Industria de la Electrónica, las Telecomunicaciones y la Informática (CANIETI).

El marco muestral se ha realizado a partir de un listado revisado directamente de los portales web de cada una de estas organizaciones, actualizado en julio de 2012, después de consultar y depurar los datos localizados. El diseño de la investigación ha respondido a un muestreo aleatorio estratificado, utilizando como criterios de diferenciación el tamaño de la empresa y el giro principal de la misma.

El número total de entrevistas se calculó según la fórmula correspondiente a este tipo de muestreo sobre la población total, de forma proporcional a los estratos fijados y con unos niveles de variabilidad de $\mathrm{p}=\mathrm{q}=0,5$, de confianza del $98 \%$ y un error del $9.20 \%$, para $\mathrm{Z}=1.96$ (Fernández Nogales, 1998). De esta manera, el tamaño de muestra estimado se refleja en la tabla 4.

\section{Operacionalización de las variables de estudio}

En la tabla 5 se definen de manera conceptual y operacional las variables de estudio de esta investigación. Primeramente se establece que la inteligencia de negocios será considerada como el centro del estudio, y por consiguiente es importante resaltar que se encuentra definida a su vez por elementos que componen una concentración del estudio de las mejores prácticas hacia el interior de las organizaciones, principalmente aquellos donde la tecnología es base de las actividades del negocio.

Estas definiciones se consideran adecuadas debido al análisis empírico del entorno de negocios dinámicos en los que se encuentran inmersas las compañías de base tecnológica y pertenecientes al sector de tecnologías de información y comunicación. En este sentido, la variable inteligencia 
Tabla 4

Determinación de la muestra

\begin{tabular}{ll}
\hline \multicolumn{2}{c}{ Población Finita: } \\
\hline Población $(\mathrm{n})$ & 161 \\
Origen de las empresas & CANIETI $^{\mathrm{a}}$ ITBaja $^{\mathrm{b}}$ \\
$\mathrm{P}$ & $50.00 \%$ \\
$\mathrm{Q}$ & $50.00 \%$ \\
Error permisible $(\mathrm{E})$ & $9.20 \%$ \\
$\mathrm{~S}$ & 1.96 \\
Nivel de confianza $(\mathrm{K})$ & $98.00 \%$ \\
Tamaño de Muestra $(\mathrm{n})=$ & 67 \\
$\mathrm{E}=2 *$ raíz $(\mathrm{N}-\mathrm{n}) /(\mathrm{n}-1) *(\mathrm{p} * \mathrm{q} / \mathrm{n})=$ & $9.39 \%$ \\
\hline
\end{tabular}

Fuente: elaboración propia.

a Cámara Nacional de la Industria de la Electrónica, Telecomunicaciones e Informática, A.C.

b Clúster de Tecnologías de Información de Baja California, A.C.

Tabla 5

Operacionalización y dimensionamiento de inteligencia de negocios

\begin{tabular}{lll}
\hline Dimensión & Definición conceptual & Definición operacional \\
\hline Sistemas de información & $\begin{array}{l}\text { Elementos electrónicos del manejo de la } \\
\text { información. Generación de } \\
\text { conocimiento a partir de la información } \\
\text { almacenada }\end{array}$ & Sistemas de información \\
& $\begin{array}{l}\text { Procesos de mejora continua } \\
\text { Tecnológicos, operacionales, } \\
\text { administrativos y estratégicos } \\
\text { Innovación }\end{array}$ & Políticas de trabajo \\
Proceso de toma de decisiones & $\begin{array}{l}\text { Metogía para toma de decisiones } \\
\text { Mecanismos, documentos, procesos y }\end{array}$ & \begin{tabular}{l} 
políticas \\
\hline
\end{tabular}
\end{tabular}

Fuente: elaboración propia.

de negocios (Business Intelligence) se subdivide en 3 dimensiones: sistemas de información, innovación y proceso de toma de decisiones.

\section{Resultados}

Los resultados de la investigación se plantean a partir de ambas perspectivas iniciales, la cualitativa y la cuantitativa. En ambos casos se llega a conclusiones que determinan el constructo sobre la inteligencia de negocios y su influencia en el desarrollo competitivo.

\section{Fase cualitativa}

Durante el proceso del estudio se llevaron a cabo una serie de actividades para realizar las entrevistas no estructuradas como parte de la investigación cualitativa. Estas actividades fueron llevadas a cabo como un esfuerzo para conocer las opiniones que los dirigentes y agentes clave de empresas de base tecnológica en la región de Tijuana, B.C. Para esta actividad se buscó entrevistar al mismo número de empresas que se contactaron en la fase cuantitativa; sin embargo, se tuvo 
Tabla 6

Principales hallazgos cualitativos

\begin{tabular}{llllll}
\hline Variable & Principales hallazgos & Número & $\%$ & $\begin{array}{l}\text { Nivel } \\
\text { competitividad }\end{array}$ & Importancia \\
\hline $\begin{array}{cllll}\text { Inteligencia de } \\
\text { negocios }\end{array}$ & Cambio cultural & 14 & 100 & Meta & $\begin{array}{l}\text { A partir de estas } \\
\text { apreciaciones se puede incidir } \\
\end{array}$ \\
& Planeación estratégica & 14 & 100 & Micro & en el impulso de políticas \\
& Innovación y calidad & 14 & 100 & Meta-Micro & públicas para el mejoramiento \\
& Propiedad intelectual & 12 & 86 & Meso & del entorno para las empresas \\
& Especialización & 10 & 72 & Meso & de base tecnológica \\
& Innovación y desarrollo & 10 & 72 & Meta-Micro & \\
& Transferencia tecnológica & 8 & 57 & Meta-Macro & \\
& Responsabilidad ambiental & 8 & 57 & Meta-Macro & \\
& Mercado internacional & 7 & 50 & Macro & \\
& Centro de desarrollo de pruebas & 7 & 50 & Micro-Macro & \\
\hline
\end{tabular}

Fuente: elaboración propia.

Tabla 7

Estudio de confiabilidad, alfa de Cronbach

\begin{tabular}{llllll}
\hline N. $^{\circ}$ & Variable & Dimensión & Nombre & No. de ítems & Alfa de Cronbach \\
\hline 1 & Competitividad & & COMPETITIVIDAD & 13 & 0.895 \\
2 & Inteligencia de negocios & Sistemas de información & BISISTEMAS & 9 & 0.925 \\
3 & & Innovación & BIINNOVACIÓN & 8 & 0.911 \\
4 & & Toma de decisiones & BIDECISIÓN & 8 & 0.932 \\
\hline
\end{tabular}

Fuente: elaboración propia.

solamente acceso a 14 de ellas, que son las que por medio de la entrevista no estructurada se obtuvo la información aquí reflejada.

Es mediante el análisis de constructo que surge a través de las entrevistas realizadas que se pueden determinar cuáles son los principales factores que inciden en la competitividad, y en este caso se muestran de manera individual en los contenidos de la tabla 6 enlistados de manera independiente.

En la tabla 6 se observa la importancia que las empresas dan a los procesos de innovación, desde el tema de cambio cultural, planeación estratégica e innovación para el desarrollo, que fue mencionado en el $100 \%$ de las empresas, hasta la relevancia de la propiedad intelectual, la especialización y la innovación y desarrollo (como proceso de investigación), hasta los aspectos de responsabilidad y orientación de mercado. Esta información corrobora que los cambios son parte de las acciones sustentadas en el conocimiento y en la implementación de procesos que gestionen su implementación y resultados (Medellín Cabrera, 2010).

\section{Fase cuantitativa}

En el instrumento utilizado se puede observar que al realizar el análisis de confiabilidad de los resultados de la aplicación del cuestionario se obtienen elevados índices de alfa de Cronbach, lo que indica que la confiabilidad del instrumento es significativa, datos que se reflejan en la tabla 7. También se puede observar que la relación entre factores y alfa de Cronbach indica que el instrumento tiene validez de contenido significativo. 
Tabla 8

ANOVA inteligencia de negocios vs competitividad

\begin{tabular}{|c|c|c|c|c|c|}
\hline & Suma de cuadrados & df & Media cuadrática & $\mathrm{F}$ & Sig. \\
\hline \multicolumn{6}{|c|}{ Inteligencia de negocios } \\
\hline Intragrupos & 32810.580 & 37 & 886.772 & 2.695 & .004 \\
\hline Intergrupos & 9542.167 & 29 & 329.040 & & \\
\hline Total & 42352.746 & 66 & & & \\
\hline Total & 13969.672 & 66 & & & \\
\hline
\end{tabular}

Fuente: elaboración propia.

En la tabla 7 se indican los componentes de cada variable, sus ítems o preguntas del cuestionario y el nivel de confiabilidad medido por el alfa de Cronbach. En la variable de estudio «competitividad» se seleccionaron 13 ítems, y en la variable «inteligencia de negocios» se utilizaron 25 ítems, divididos en 3 dimensiones: sistemas de información con, 9 ítems; innovación, con 8 ítems, y toma de decisiones, con 8 ítems.

En total se implementó un cuestionario de 38 ítems. La confiabilidad se mantiene muy elevada para las 4 dimensiones ilustradas. El diseño del dicho cuestionario se llevó a cabo tomando en consideración cada una de las dimensiones y subsecuentes indicadores arrojados y sus variables correspondientes, tanto dependientes como independientes, además de llevar a cabo las pruebas estadísticas pertinentes sobre el propio instrumento una vez elaborado, tal y como se muestra en el Anexo I.

Se desarrolla el análisis ANOVA para encontrar la relación entre las variables predictoras y la variable dependiente, como se explica en la tabla 8.

Observaciones. Se observa una relación directa positiva presente entre la competitividad y su variable predictora, inteligencia de negocios. Se concluye que tales acciones de fortalecimiento deben seguir y mejorar en lo posible (fig. 12). Los resultados del análisis de varianza indican que la variable predictora tienen un efecto significante en la competitividad. En el caso de inteligencia de negocios, donde $\mathrm{F}(37,29)=2.695$ y $\mathrm{p}=0.004$, las media general de 90.4925 presenta una tendencia hacia el incremento de la competitividad en relación a inteligencia de negocios.

\section{Correlación bivariada}

A continuación se procede a describir las correlaciones que existen entre las variables de estudio. La figura 13 muestra la correlación existente y analizada en este instrumento, donde existe una relación lineal entre las variables inteligencia de negocios [Business Intelligence] y competitividad. La figura también muestra que existe una relativa homocedasticidad debido a que las lecturas mantienen una dispersión similar entre los puntos de recorrido.

\section{Correlación de Pearson momento-producto}

En la tabla 9 se observa una alta correlación entre competitividad y Business Intelligence $(\mathrm{r}=0.737$ y $\mathrm{p}<0.001)$. Esta interpretación no implica causalidad; la relación significante solamente implica que las variables covarían entre ellas si existe relación entre las variables 


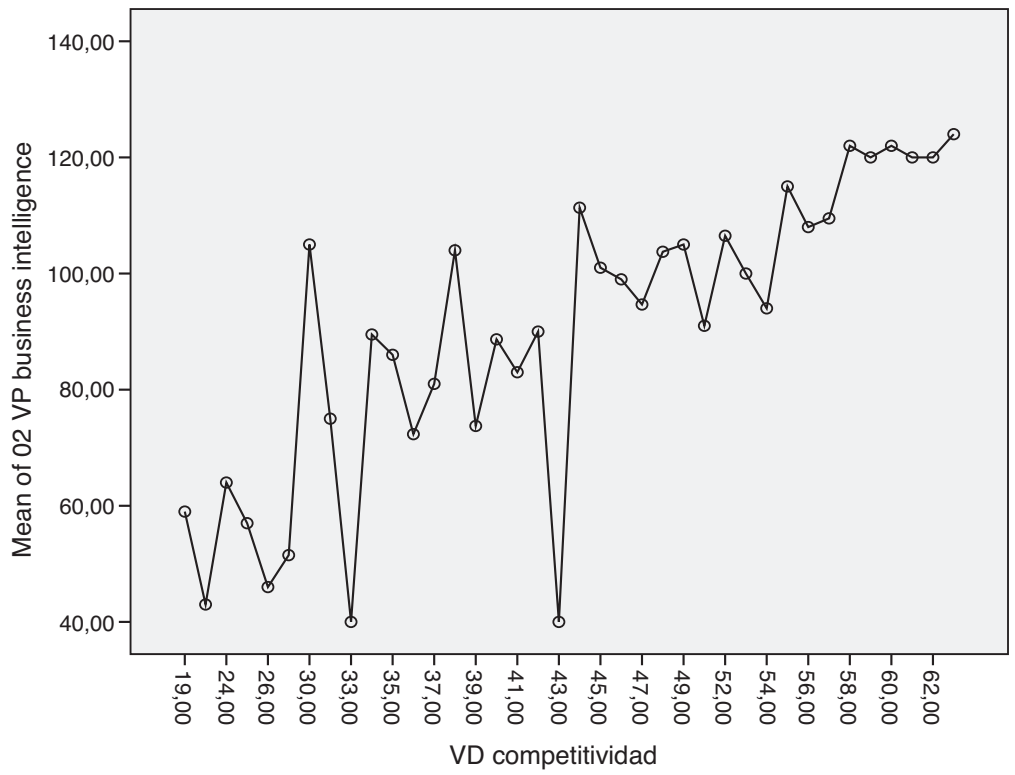

Figura 12. ANOVA de competitividad en términos de variables predictoras.

Fuente: elaboración propia.

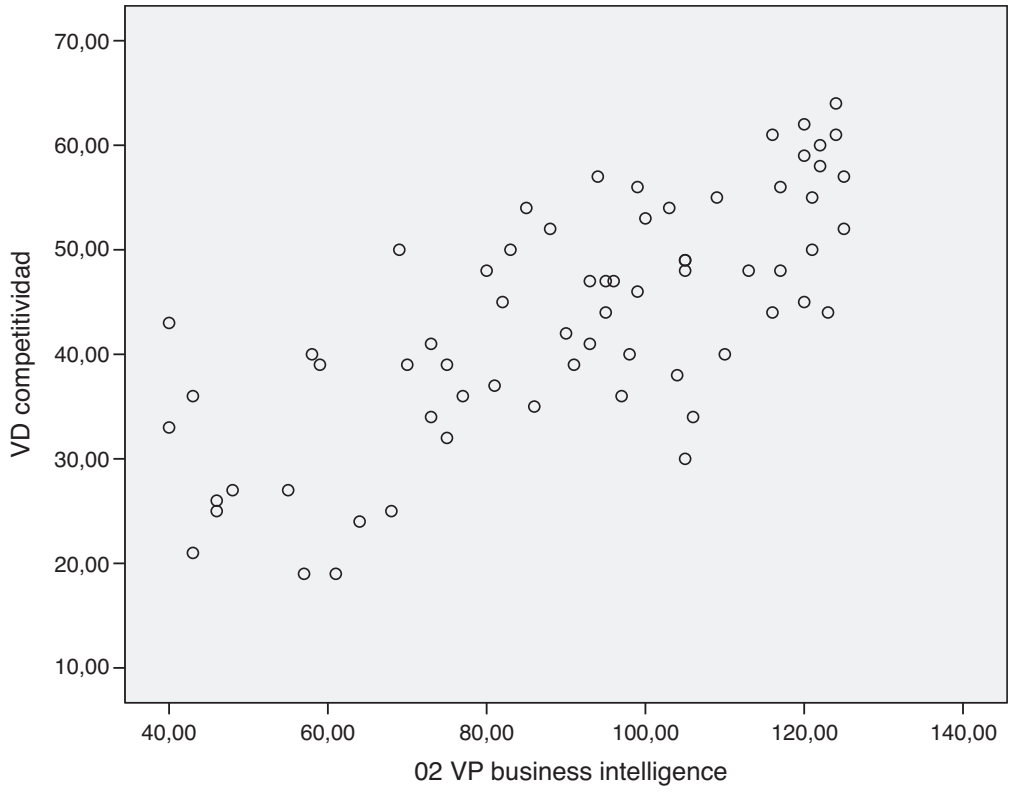

Figura 13. Gráfico de correlación de competitividad vs Business Intelligence.

Fuente: elaboración propia. 
Tabla 9

Tabla de correlación de Pearson

\begin{tabular}{lll}
\hline & Competitividad & Inteligencia de negocios \\
\hline $\begin{array}{l}\text { Competitividad } \\
\text { Correlación de Pearson }\end{array}$ & 1 & \\
$\quad \begin{array}{l}\text { Sig. (bilateral) } \\
\mathrm{N}\end{array}$ & 67 & 1 \\
$\begin{array}{l}\text { Inteligencia de negocios } \\
\quad \text { Correlación de Pearson }\end{array}$ & $0.737^{* *}$ & \\
$\quad$ Sig. (bilateral) & 0.000 & 67 \\
$\mathrm{~N}$ & 67 & \\
\hline
\end{tabular}

Fuente: elaboración propia.

** La correlación es significativa al nivel 0.01 (bilateral).

Tabla 10

Tabla de correlación de Spearman

\begin{tabular}{lll}
\hline & Competitividad & Inteligencia de negocios \\
\hline $\begin{array}{l}\text { Rho de Spearman } \\
\text { Competitividad } \\
\text { Coeficiente de correlación }\end{array}$ & 1.000 & \\
$\quad$ Sig. (bilateral) &. & \\
N & 67 & 1.000 \\
Inteligencia de negocios & &. \\
Coeficiente de correlación & $0.720^{* *}$ & 67 \\
Sig. (bilateral) & 0.000 & \\
$\mathrm{~N}$ & 67 & \\
\hline
\end{tabular}

Fuente: Elaboración propia.

** La correlación es significativa al nivel 0.01 (bilateral).

predictoras y la variable dependiente mayor a 0.500 y con un nivel de significación menor a 0.01 .

\section{Correlación de Spearman}

En la tabla 10, el rango del coeficiente de Spearman obtenido entre las variables competitividad y Business Intelligence $(\mathrm{rho}=0.720, \mathrm{p}<0.001)$ sustenta los resultados de la correlación de Pearson si existe relación entre las variables predictoras y la variable dependiente mayor a 0.500 y con un nivel de significación menor a 0.01 .

\section{Conclusiones}

Las conclusiones que se presentan a continuación resultan del estudio realizado y constan de 2 partes:

- Fase cualitativa. Esta primera fase consistió en realizar una entrevista no estructurada a 14 empresas medianas y grandes del sector de tecnologías de información en el estado de Baja California, todas ellas con alguna relación comercial, laboral, de vinculación o cualquier otra que tenga injerencia en el desarrollo económico de la ciudad de Tijuana, B.C. 


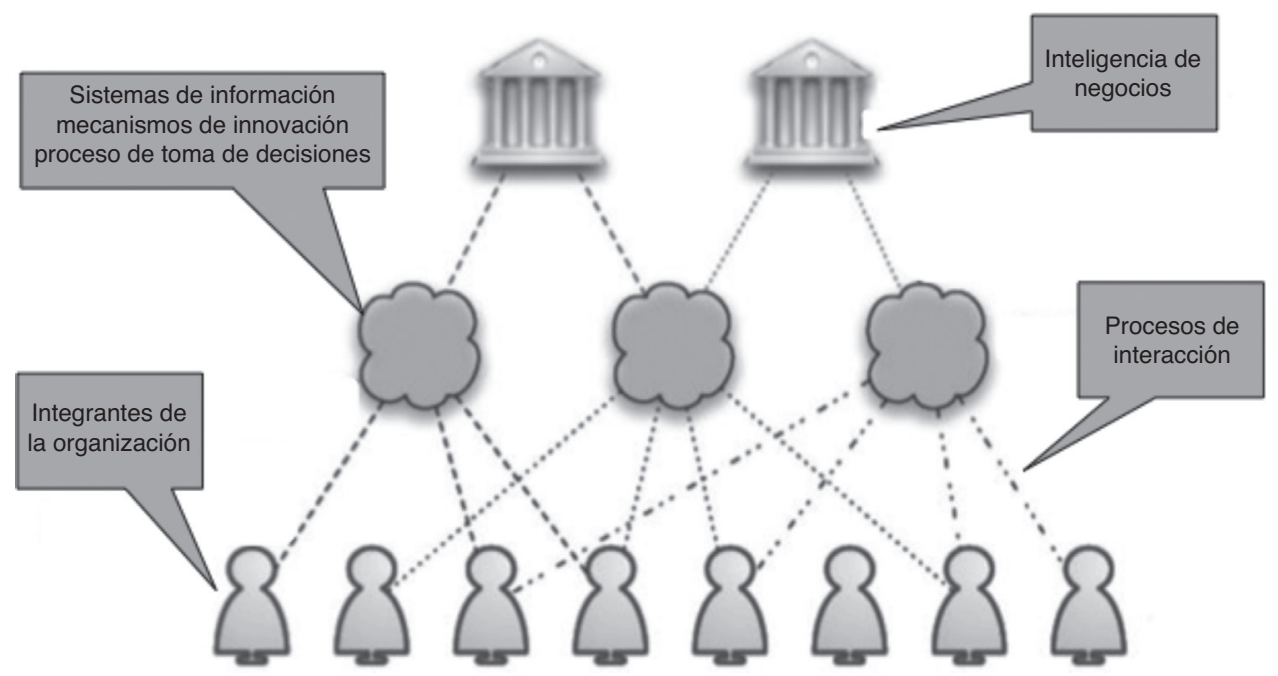

Figura 14. Arquitectura del estudio de inteligencia de negocio y adecuación para estudios posteriores. Fuente: elaboración propia.

- Fase cuantitativa. En esta fase se aplicaron un total de 67 cuestionarios a igual número de empresas, de las cuales 23 fueron micros, 18 pequeñas, 3 medianas y 23 grandes empresas del sector de tecnologías de la información en la ciudad de Tijuana, B.C. Las variables bajo estudio se analizaron utilizando las técnicas estadísticas de alfa de Cronbach, ANOVA, correlación de Pearson y correlación de Spearman.

Ambas fases permitieron cumplir el objetivo planteado al inicio de este trabajo, además de plantear un enfoque adecuado para el estudio de la inteligencia de negocios en el contexto empresarial.

1) Describir, analizar y correlacionar los sistemas de información, la innovación y los procesos de toma de decisiones como factores de incremento de inteligencia de negocios en el desarrollo de competitividad desde el enfoque de la gestión del conocimiento en las empresas de base tecnológica en Tijuana, B.C.

2) La figura 14 muestra la estructura fundamental del estudio. La base del estudio es el personal empleado profesional y perteneciente a una empresa de base tecnológica en el sector de tecnologías de información de Tijuana, B.C., así mismo, los procesos de interacción que se llevan a cabo entre ellos y las estrategias de inteligencia de negocios, que se definen como: sistemas de información; mecanismos de innovación; y el proceso de toma de decisiones. Este entorno se refiere al análisis de la inteligencia de negocios y se perfila como un entorno que se puede adecuar para su estudio posterior mediante el uso de metodologías de ciencias computacionales que ayuden a confirmar la información que se presenta y analiza de manera tradicional a través del estudio mixto. 


\section{Hallazgos en la fase cualitativa}

1. El conocimiento se presenta como uno de los activos que mayor valor pueden dar a las empresas.El estudio indica que los líderes y elementos humanos clave de las organizaciones que fueron estudiadas encuentran una referencia importante hacia el conocimiento. Desde la propiedad intelectual y la transferencia tecnológica hasta la innovación, las actividades de investigación y desarrollo, se manifiesta la importancia clave de este tipo de activos intangibles para el crecimiento de las empresas de base tecnológica que se encuentran ubicadas en la entidad bajacaliforniana.La importancia del sector de tecnologías de información se reafirma cuando este sector se actualiza e incrementa el valor de los procesos de innovación y de los elementos humanos especializados que existen en la región. Las personas y los procesos son fundamentalmente de índole logicomatemática; esto quiere decir que muchas de sus actividades productivas giran en torno al valor del conocimiento y de cómo este se manifiesta en la posibilidad de negocios para la organización.

2. El entorno de negocios es fundamentalmente una labor conjunta entre las empresas y el gobierno; sin embargo, es responsabilidad de este último otorgar las condiciones necesarias que faciliten a las empresas concretar mayor número de proyectos.La posibilidad de realizar negocios y de elevar la competitividad de las empresas es un eje que no depende enteramente de la misma organización. Para ello es importante la existencia de los medios que faciliten el acceso a los mercados nacionales e internacionales, pero al mismo tiempo que garanticen la existencia de un entorno sociopolítico y económico estable para que la región se vuelva receptora de inversión en el ramo de la investigación y desarrollo de nuevos productos.Es bajo este contexto que la inteligencia de negocios como herramienta o metodología no es una estrategia que por sí sola pueda elevar la competitividad de la organización. En este sentido, la realidad sistémica del entorno regional influye en el resultado de las acciones implementadas para mejorar la capacidad de las organizaciones de generar valor en sus productos y servicios. Este hallazgo ilustra la complejidad inherente en las empresas, donde, a pesar de que los procesos para generar inteligencia son importantes, estos no son exitosos a menos que se combinen una serie de condiciones sistémicas que componen la competitividad de los negocios.

3. La innovación, el uso de sistemas de información, así como los procesos de toma de decisión, son actividades fundamentales para el aprendizaje de las empresas.La innovación también se considera una de las vertientes que ayudan al aprendizaje de las empresas y a su fortalecimiento en el mercado de las tecnologías de la información. Para que esta estrategia empresarial tenga éxito, se debe contar con mano de obra altamente especializada, capacitada y certificada. La cultura organizacional, las políticas públicas y la vinculación con el sector educativo son fundamentales, y la razón principal para que se dependa de estas condiciones es que es necesario que se lleve a cabo un cambio cultural con un mayor enfoque en la calidad, en el desarrollo sustentable y en el respeto, así como la protección de la propiedad intelectual, todos ellos fundamentos de un entorno de innovación, investigación y desarrollo como arista tanto de un crecimiento económico como del desarrollo de la competitividad en las empresas del sector.

Las variables de estudio forman parte del constructo social en el cual se ubican las empresas de Tecnologías de Información (TI). Los procesos de inteligencia de negocios intervienen directamente en la apreciación que se tiene de la competitividad en las organizaciones. Áreas de oportunidad que siempre están una continua mejoría son la vinculación, la transferencia 
tecnológica y la especialización del capital humano por medio de procesos educativos certificados que mejoren las opciones de generar valor y propiedad intelectual para las organizaciones.

\section{Hallazgos en la fase cuantitativa}

1. Se valida la hipótesis que se refiere a la relación positiva que existe entre la inteligencia de negocios y el incremento de la competitividad en las empresas de base tecnológica de la ciudad de Tijuana, B.C.En esta conclusión se verifica que la variable interviene directamente de forma positiva en el incremento de la competitividad. La evaluación de los datos obtenidos de manera empírica con el análisis estadístico llevado a cabo mediante el software SPSS confirman una relación positiva de la variable predictora con la variable dependiente.

2. Los procesos de inteligencia de negocios son parte de la generación de competitividad y su importancia se manifiesta en el enfoque de la gestión del conocimiento en las empresas de base tecnológica de la ciudad de Tijuana, B.C.La variable «inteligencia de negocios» está en los resultados de investigación cuantitativa.

En resumen, se concluye que la investigación tiene contribuciones para el estudio de la gestión del conocimiento y su influencia en la competitividad por lo siguiente:

1. Aporta evidencia empírica sobre los procesos de inteligencia de negocios como fases o variables del proceso que genera competitividad en empresas del sector de tecnologías de información o de base tecnológica. Este estudio analiza estas vertientes por su enfoque sistémico.

2. Combina métodos de investigación al emplear la lógica deductiva e inductiva para la construcción del modelo de competitividad desde el enfoque de gestión del conocimiento con la finalidad de analizar la inteligencia de negocios en las empresas de base tecnológica. Los resultados de la investigación cualitativa proporcionan una base confiable para la conceptualización y operacionalización del modelo, así como también ofrecen información útil para la construcción del instrumento de medición que da pie al análisis estadístico de la investigación cuantitativa. Como parte de la continuidad de esta investigación se pretende implementar métodos estadísticos y computacionales para incrementar la validez de los resultados obtenidos. La intención de triangulación de métodos provee a esta investigación conclusiones con mayor sustento metodológico.

3. Se analiza el proceso de generación de competitividad desde un enfoque de gestión del conocimiento que toma como referencia las áreas organizacionales de la empresa relacionadas con la inteligencia de negocios. Estos procesos, mediante la implementación de políticas organizacionales tales como las actividades de innovación, el uso de sistemas de información y los procesos de toma de decisiones, aportan al desarrollo de la competitividad.

4. Se propone la modificación de la estrategia en materia de fortalecimiento de la competitividad en las empresas del sector de TI, reenfocando el apoyo hacia la certificación de los empleados a una mejora de los procesos de inteligencia de negocios

\section{Implicaciones teóricas y prácticas}

Este estudio presenta implicaciones significativas tanto para la teoría como para la práctica. Las implicaciones teóricas van más allá de una simple comprensión de los factores que facilitan la implementación de la inteligencia de negocios como un eje fundamental en la generación de competitividad en las empresas de un determinado sector. En un entorno dinámico de negocios 
se observa la necesidad de mayor literatura que relacione de manera holística y sistémica los esfuerzos sociales que se llevan a cabo para generar competitividad empresarial mediante la implementación de estrategias de inteligencia hacia la empresa como una entidad independiente, con sus propias características, habilidades, valores, conocimientos, conductas y capacidades (Bueno, 2008).

Mediante la orientación planteada en este estudio se propone una nueva visión a través de la variable predictora utilizada, que se refiere a los retos que tienen las empresas que compiten en entornos turbulentos y con una gran necesidad de tomar acciones innovadores, de calidad y de constante actualización técnica, como en este caso lo es la inteligencia de negocios que puede variar para otros sectores y empresas (Carrillo et al., 2012).

Por otra parte, el uso de los métodos de investigación mixtos fortalece el desarrollo del proceso indagatorio (Hernández Sampieri, Fernández Collado y Baptista Lucio, 2014). Esto abre la posibilidad para realizar el estudio de situaciones utópicas donde se pueden evaluar los indicadores de cada uno de los elementos que conllevan una modificación en el desarrollo de competitividad, además del uso de la inteligencia de negocios (Larson, 2009).

\section{Limitaciones de la investigación}

La presente investigación proporciona un modelo conceptual de cómo las empresas del sector de tecnologías de información o de base tecnológica desarrollan su competitividad desde un enfoque de gestión del conocimiento aplicando la inteligencia de negocios para ello. Este modelo ha sido probado empíricamente a través de la investigación cuantitativa utilizando las técnicas estadísticas mencionadas anteriormente. En este contexto, surgen algunas limitaciones en los siguientes rubros:

\section{Recopilación de los datos}

La aplicación de los cuestionarios fue de forma aleatoria siguiendo la cantidad muestral obtenida mediante el uso de las fórmulas estadísticas aplicables a este caso. La identificación de los empleados clave para obtener información confiable de las empresas fue de forma cualitativa y considerando el puesto y la antigüedad del mismo dentro de la organización.

Es importante resaltar que existe una variación considerable entre las empresas en cuanto a sus estrategias competitivas y a su implementación sobre inteligencia de negocios. El tamaño de la empresa es un factor determinante en los temas de sistemas de información, innovación y procesos de toma de decisiones. Aun a pesar de las diferencias indicadas, los resultados de la investigación no demuestran errores graves en la planeación estratégica de las empresas del sector. En un futuro es conveniente replicar el estudio a empresas más homogéneas.

\section{Generalización de resultados}

Dada la especificidad del presente estudio sobre empresas del sector de TI, las cuales por su naturaleza operan en entornos dinámicos, se puede inferir que es probable que las empresas que operan en entornos similares tengan los mismos elementos contextuales para generar competitividad. Sin embargo, no se pueden generalizar los resultados de este estudio a empresas que operan en entornos estables, puesto que no se ha estudiado este tipo de organizaciones. Una investigación de tipo cross sectional entre múltiples sectores industriales aportaría una mejor comprensión de las dinámicas de generar competitividad. 
Lineas de investigación y trabajo futuro

El trabajo conceptual y empírico en esta área del conocimiento se encuentra en una etapa inicial, y las contribuciones de la investigación sugieren algunas líneas de investigación para futuros enfoques de análisis. Algunas de ellas son las siguientes:

1. La implementación de estrategias de inteligencia de negocios para detonar la generación de competitividad en organizaciones que operan en entornos dinámicos o estables desde un enfoque de gestión del conocimiento se deben adecuar a cada sector industrial.

2. Esta investigación realiza una relación somera de las variables de estudio, de las teorías de competitividad y de la inteligencia de negocios; es importante continuar con el estudio de esta relación para fortalecer los resultados obtenidos en este estudio.

3. Este estudio se refiere a la generación de competitividad partiendo de la gestión del conocimiento; es importante validar los resultados desde un enfoque financiero que estudie más detenidamente las implicaciones en la rentabilidad de la empresa.

\section{Anexo I. Análisis del instrumento de medición}

\section{A.1. Descripción de la estructura del cuestionario}

La variable predictiva o dependiente «competitividad» se dimensiona en una categoría y la variable predictora o independiente «Business Intelligence» se dimensiona en 3 categorías; estas a su vez se derivan en 3 indicadores, y a cada indicador se le desarrollaron 3 ítems o preguntas.

(tabla A1)

(tabla A2)

(tabla A3)

\section{A.2. Análisis de confiabilidad, variable «competitividad»}

De acuerdo con Nunnally (1967), los valores de alfa de Cronbach entre 0.6 y 0.7 son aceptables para ítems que han de formar un mismo constructo. El resultado de 0.895 nos indica una alta consistencia interna entre los ítems de competitividad.

Tabla A1

Ítems de la variable predictora Business Intelligence y sus dimensiones

\begin{tabular}{llll}
\hline $\begin{array}{l}\text { Variable } \\
\text { Competitividad }\end{array}$ & $\begin{array}{l}\text { Dimensión } \\
\text { Competitividad }\end{array}$ & Indicadores & $\begin{array}{c}\text { Ítems } \\
13\end{array}$ \\
\hline $\begin{array}{l}\text { Inteligencia de } \\
\text { negocios (Business }\end{array}$ & Sistemas de & Operativo & 3 \\
Intelligence) & información & Estrategia & 3 \\
& & Base de datos & 3 \\
& Innovación & Investigación y desarrollo & 2 \\
& & Nuevos productos & 3 \\
& Proceso de toma de & Nuevos procesos & 3 \\
& decisiones & Estructura organizacional & 3 \\
& & Sistemas de calidad & 2 \\
& & La gerencia & 2
\end{tabular}

Fuente: elaboración propia. 
Tabla A2

Confiabilidad de la Variable Dependiente «Competitividad

\begin{tabular}{lll}
\hline Alfa de Cronbach & Estadísticos de fiabilidad & N de elementos \\
\hline 0.895 & 13
\end{tabular}

Estadísticos total-elemento

\begin{tabular}{|c|c|c|c|c|}
\hline & $\begin{array}{l}\text { Media de la } \\
\text { escala si se } \\
\text { elimina el } \\
\text { elemento }\end{array}$ & $\begin{array}{l}\text { Varianza de la } \\
\text { escala si se } \\
\text { elimina el } \\
\text { elemento }\end{array}$ & $\begin{array}{l}\text { Correlación } \\
\text { elemento-total } \\
\text { corregida }\end{array}$ & $\begin{array}{l}\text { Alfa de } \\
\text { Cronbach si se } \\
\text { elimina el } \\
\text { elemento }\end{array}$ \\
\hline $\begin{array}{l}\text { p1 Las certificaciones nos han ayudado a } \\
\text { tener más clientes }\end{array}$ & 37.00 & 128.000 & 0.505 & 0.893 \\
\hline $\begin{array}{l}\text { p2 Un alto porcentaje de ingresos proviene } \\
\text { de los proyectos que hemos desarrollado } \\
\text { en equipo con otras empresas }\end{array}$ & 37.40 & 127.145 & 0.677 & 0.884 \\
\hline $\begin{array}{l}\text { p3 Se han desarrollado procesos y } \\
\text { mecanismos para mejorar la relación con } \\
\text { los proveedores y han ayudado a } \\
\text { incrementar las ventas }\end{array}$ & 36.83 & 120.902 & 0.781 & 0.878 \\
\hline $\begin{array}{l}\text { p4 Nuestra clientes nos buscan para } \\
\text { brindarles más productos y/o servicios }\end{array}$ & 36.10 & 132.714 & 0.530 & 0.891 \\
\hline $\begin{array}{l}\text { p5 En esta empresa se valora la capacidad } \\
\text { creativa de los empleados y esto se ve } \\
\text { como una ventaja sobre la competencia }\end{array}$ & 36.23 & 129.357 & 0.653 & 0.885 \\
\hline $\begin{array}{l}\text { p6 La calidad de nuestros productos y/o } \\
\text { servicios nos da ventaja sobre la } \\
\text { competencia }\end{array}$ & 36.00 & 134.897 & 0.441 & 0.894 \\
\hline $\begin{array}{l}\text { p7 La cultura de la región está enfocada en la } \\
\text { creación de conocimiento y se valora a las } \\
\text { personas que se dedican a ello }\end{array}$ & 36.63 & 128.309 & 0.729 & 0.883 \\
\hline $\begin{array}{l}\text { p8 Las empresas de este sector autorregulan } \\
\text { el conocimiento que generan buscando } \\
\text { patentes y promoviendo acciones } \\
\text { empresariales para mejorar su } \\
\text { competitividad }\end{array}$ & 36.93 & 128.478 & 0.613 & 0.887 \\
\hline $\begin{array}{l}\text { p9 Se cuenta con apoyo gubernamental para } \\
\text { mejorar el desempeño de esta empresa } \\
\text { dentro de su sector económico }\end{array}$ & 37.50 & 128.121 & 0.604 & 0.887 \\
\hline $\begin{array}{l}\text { p10 La estabilidad económica, el control de } \\
\text { la corrupción y la efectividad del gobierno } \\
\text { regional son factores que ayudan a } \\
\text { mejorar la competitividad de esta empresa }\end{array}$ & 37.10 & 125.059 & 0.639 & 0.886 \\
\hline $\begin{array}{l}\text { p11 Nuestra empresa utiliza o ha utilizado } \\
\text { apoyos gubernamentales para mejorar su } \\
\text { competitividad }\end{array}$ & 37.37 & 129.344 & 0.502 & 0.893 \\
\hline $\begin{array}{l}\text { p12 Esta empresa busca continuamente } \\
\text { aprovechar las ventajas de los apoyos que } \\
\text { el gobierno destina a las empresas de este } \\
\text { sector económico }\end{array}$ & 37.43 & 127.495 & 0.600 & 0.887 \\
\hline $\begin{array}{l}\text { P13 La estabilidad económica de la región } \\
\text { estimula la competitividad de nuestra } \\
\text { empresa }\end{array}$ & 36.67 & 133.057 & 0.506 & 0.892 \\
\hline
\end{tabular}

Fuente: elaboración propia. 
Tabla A3

Confiabilidad de la variable predictora «Business Intelligence»: «sistemas de información»

Estadísticos de fiabilidad

Alfa de Cronbach

$\mathrm{N}$ de elementos

0.925

9

Estadísticos total-elemento

\begin{tabular}{|c|c|c|c|c|}
\hline & $\begin{array}{l}\text { Media de la } \\
\text { escala si se } \\
\text { elimina el } \\
\text { elemento }\end{array}$ & $\begin{array}{l}\text { Varianza de la } \\
\text { escala si se } \\
\text { elimina el } \\
\text { elemento }\end{array}$ & $\begin{array}{l}\text { Correlación } \\
\text { elemento-total } \\
\text { corregida }\end{array}$ & $\begin{array}{l}\text { Alfa de } \\
\text { Cronbach si se } \\
\text { elimina el } \\
\text { elemento }\end{array}$ \\
\hline $\begin{array}{l}\text { p14 En esta empresa tenemos sistemas } \\
\text { computacionales para registrar las actividades } \\
\text { productivas y/o de ventas }\end{array}$ & 27.73 & 82.064 & 0.837 & 0.910 \\
\hline $\begin{array}{l}\text { p15 En esta empresa se cuenta con sistemas que } \\
\text { ayudan a controlar las actividades productivas de } \\
\text { los empleados }\end{array}$ & 28.20 & 81.407 & 0.713 & 0.918 \\
\hline $\begin{array}{l}\text { p16 En esta empresa se manejan las actividades } \\
\text { diarias por medio de sistemas de información } \\
\text { computacional }\end{array}$ & 27.80 & 80.303 & 0.779 & 0.913 \\
\hline $\begin{array}{l}\text { p17 Existen sistemas que ayudan a predecir los } \\
\text { requerimientos de producción para así estar listos } \\
\text { ante cualquier eventualidad del mercado }\end{array}$ & 28.57 & 84.392 & 0.719 & 0.917 \\
\hline $\begin{array}{l}\text { p18 En esta empresa se tienen sistemas que ayudan } \\
\text { a predecir las ventas que se realizaran en un } \\
\text { periodo determinado de tiempo }\end{array}$ & 28.43 & 82.185 & 0.757 & 0.915 \\
\hline $\begin{array}{l}\text { p19 Existen sistemas que guardan información el } \\
\text { tiempo suficiente para que ayuden a tomar } \\
\text { decisiones para el futuro de la empresa }\end{array}$ & 28.13 & 80.533 & 0.827 & 0.910 \\
\hline $\begin{array}{l}\text { p20 Contamos con una base de datos operativa del } \\
\text { negocio que recaba la información día a día en } \\
\text { cuanto a operaciones y/o actividades de } \\
\text { empleados y/o ventas }\end{array}$ & 28.00 & 80.966 & 0.752 & 0.915 \\
\hline $\begin{array}{l}\text { p21 Se cuenta con una base de conocimiento para el } \\
\text { uso de los empleados y mejora de los procesos de } \\
\text { negocio }\end{array}$ & 28.07 & 86.754 & 0.666 & 0.920 \\
\hline $\begin{array}{l}\text { p22 La información que se genera en la empresa se } \\
\text { guarda durante un tiempo razonable para } \\
\text { consultarla en el momento que se requiera y se } \\
\text { mantiene respaldo de ella }\end{array}$ & 27.60 & 92.662 & 0.511 & 0.928 \\
\hline \multicolumn{5}{|c|}{ Estadísticos de la escala } \\
\hline Varianza & \multicolumn{2}{|c|}{ Desviación estándar } & \multicolumn{2}{|c|}{$\mathrm{N}$ de elementos } \\
\hline 104.668 & \multicolumn{2}{|l|}{10.231} & \multicolumn{2}{|l|}{9} \\
\hline
\end{tabular}

Fuente: elaboración propia.

\section{A.2.1. Variable «Business Intelligence»: dimensión «sistemas de información»}

El resultado de 0.925 nos indica alta consistencia interna entre los ítems de sistemas de información; se detecta que el ítem p36 muestra un incremento a 0.928 en el nivel de confiabilidad, pero se considera que no es relevante el cambio, por lo que el cuestionario queda de la misma forma. 
Tabla A4

Confiabilidad de la variable predictora «Business Intelligence»: «innovación»

Estadísticos de fiabilidad

\begin{tabular}{lc}
\hline Alfa de Cronbach & N de elementos \\
\hline 0.911 & 8
\end{tabular}

Estadísticos total-elemento

\begin{tabular}{|c|c|c|c|c|}
\hline & $\begin{array}{l}\text { Media de la } \\
\text { escala si se } \\
\text { elimina el } \\
\text { elemento }\end{array}$ & $\begin{array}{l}\text { Varianza de la } \\
\text { escala si se } \\
\text { elimina el } \\
\text { elemento }\end{array}$ & $\begin{array}{l}\text { Correlación } \\
\text { elemento-total } \\
\text { corregida }\end{array}$ & $\begin{array}{l}\text { Alfa de } \\
\text { Cronbach si se } \\
\text { elimina el } \\
\text { elemento }\end{array}$ \\
\hline $\begin{array}{l}\text { p23 Esta empresa destina una cantidad } \\
\text { presupuestal para desarrollar nuevos } \\
\text { productos y/o servicios }\end{array}$ & 22.93 & 58.271 & 0.521 & 0.917 \\
\hline $\begin{array}{l}\text { p24 En esta empresa se me impulsa a desarrollar } \\
\text { actividades que generen nuevos productos y/o } \\
\text { servicios }\end{array}$ & 22.60 & 57.352 & 0.727 & 0.899 \\
\hline $\begin{array}{l}\text { p25 Esta empresa fundamenta su crecimiento en } \\
\text { el análisis, desarrollo e implementación de } \\
\text { nuevos productos }\end{array}$ & 22.60 & 55.214 & 0.739 & 0.897 \\
\hline $\begin{array}{l}\text { p26 Una considerable parte del tiempo de } \\
\text { trabajo diario se dedica a la planeación de } \\
\text { nuevos productos }\end{array}$ & 23.27 & 57.926 & 0.727 & 0.899 \\
\hline $\begin{array}{l}\text { p27 Son los nuevos productos lo que nos } \\
\text { diferencia de nuestra competencia }\end{array}$ & 23.00 & 53.172 & 0.796 & 0.892 \\
\hline $\begin{array}{l}\text { p28 Esta empresa considera que es importante } \\
\text { mejorar y agilizar los procesos de negocio de } \\
\text { una forma continua }\end{array}$ & 22.27 & 55.582 & 0.785 & 0.894 \\
\hline $\begin{array}{l}\text { p29 Esta empresa busca formas certificadas de } \\
\text { llevar a cabo una mejora continua de sus } \\
\text { procesos de negocio }\end{array}$ & 22.73 & 53.995 & 0.784 & 0.893 \\
\hline $\begin{array}{l}\text { p30 En nuestra empresa se busca la certificación } \\
\text { de los procesos y esto nos brinda una ventaja } \\
\text { sobre nuestros competidores }\end{array}$ & 22.60 & 56.800 & 0.653 & 0.905 \\
\hline \multicolumn{5}{|c|}{ Estadísticos de la escala } \\
\hline Varianza & \multicolumn{2}{|c|}{ Desviación estándar } & \multicolumn{2}{|c|}{$\mathrm{N}$ de elementos } \\
\hline 72.276 & \multicolumn{2}{|l|}{8.502} & \multicolumn{2}{|c|}{8} \\
\hline
\end{tabular}

Fuente: Elaboración propia.

\section{A.2.2. Variable «Business Intelligence»: dimensión «innovación»}

(tablas A4 y A5)

El resultado de 0.911 nos indica alta consistencia interna entre los ítems de innovación; se detecta que el ítem p37 muestra un incremento a 0.917 en el nivel de confiabilidad, pero se considera que no es relevante el cambio, por lo que el cuestionario queda de la misma forma.

\section{A.2.3. Variable «Business Intelligence»: dimensión «proceso de toma de decisiones»}

El resultado de 0.932 nos indica alta consistencia interna entre los ítems de proceso de toma de decisiones; esta es la confiabilidad más alta que presenta el análisis, por lo que el cuestionario queda de la misma forma. 
Tabla A5

Confiabilidad de la Variable Predictora «Business Intelligence»: «Toma de Decisiones»

Estadísticos de fiabilidad

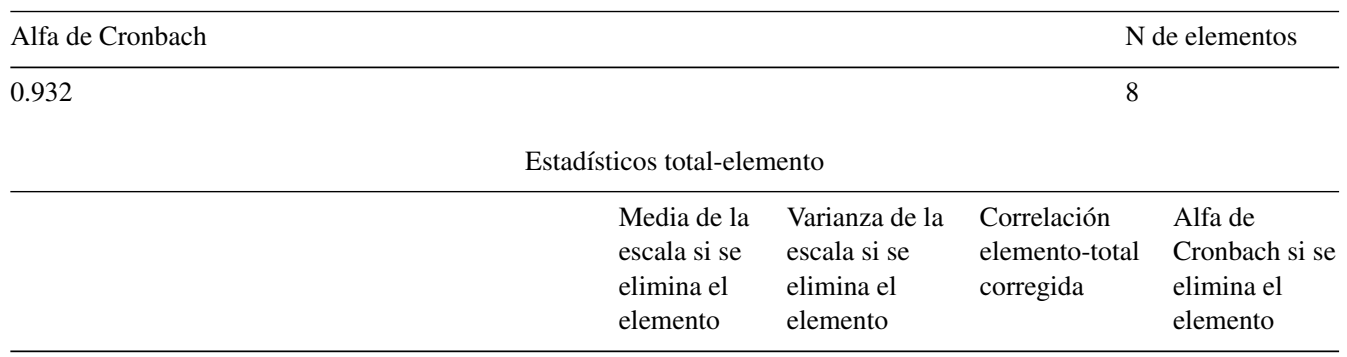

p31 Se cuenta con una estructura organizacional y

24.47

58.464

0.811

0.920

con la descripción de funciones de cada uno de

los puestos que el organigrama presenta, todos los empleados lo conocen

p32 Los equipos de trabajo son diversos y las

0.717

0.927

funciones de los empleados se complementan para llevar a cabo la toma de decisiones

p33 La descripción de las funciones incluye

60.161

0.887

0.915 actividades para la innovación, la investigación y desarrollo haciendo a la empresa más competitiva

p34 En esta empresa se cuenta con personal encargado de monitorear la calidad de los productos y/o servicios que proveemos

p35 La calidad es uno de los factores que mayor influencia tienen en el proceso de toma de decisiones

p36 Los procesos de toma de decisiones están sujetos a la misión y visión de la empresa

p37 Las opiniones de los empleados son tomadas en cuenta al momento de decidir las acciones estratégicas de la empresa, tales como capacitación, desarrollo de productos y/o servicios

p38 Para llegar a una toma de decisión se analizan los sistemas de información con que se cuenta en la empresa

estadísticos de la escala

\begin{tabular}{lllc}
\hline Media & Varianza & Desviación estándar & N de elementos \\
\hline 27.53 & 80.120 & 8.951 & 8 \\
\hline
\end{tabular}

Fuente: elaboración propia.

\section{Referencias}

Ahumada-Tello, E. (2011). La Gestión del Conocimiento en la Generación de Competitividad Sistémica: El Sector de Tecnologías de la Información en Tijuana, B.C. Tijuana: Universidad Autónoma de Baja California.

Ahumada-Tello, E., Zárate Cornejo, R. E., Plascencia López, I. y Perusquia-Velasco, J. M. (2012). Modelo de competitividad basado en el conocimiento: el caso de las pymes del sector de tecnologías de información en Baja California. Revista International Administración \& Finanzas, 5(4), 13-27. 
Ángel, A. C. (2010). La imposibilidad de la certeza. Debates IESA, 15(4), 11.

Araya Guzmán, S. A. (2004). Los sistemas de información y su interacción con la dimensión cultural de las organizaciones. Revista Ingeniería Industrial, 3(1), 13.

Bara, A., Botha, I., Diaconita, V., Lungu, I., Velicanu, A. y Velicanu, M. (2009). A model for business intelligence systems' development. Informatica Economica, 13(4), 99-108.

Berg de Valdivia, C. M. (2007). Gestión del conocimiento para la mejora de la competitividad de las empresas de telecomunicaciones. Lima, Perú: Universidad Nacional de Ingeniería.

Bueno, E. (2008). La Sociedad del Conocimiento: una realidad inacabada. En J. Micheli, E. Medellín, A. Hidalgo, y J. Jassó (Eds.), Conocimiento e Innovación: Retos de la Gestión Empresarial (pp. 25-55). México: UAM-UNAM-Plaza y Valdés.

Bueno, E. (2000). Perspectivas sobre dirección del conocimiento y capital intelectual. Madrid: Instituto Universitario Eurofórum Escorial.

Carrillo, J., Hualde, A. y Villavicencio, D. (2012). Dilemas de la Innovación en México. Dinámicas sectoriales, territoriales e institucionales. Tijuana, Baja California, México: El Colegio de la Frontera Norte.

Chaabouni, A. y Triki, A. (2013). Contribution of an ERP (Enterprise Resource Planning) system to the decision making: Case of two industrial SMEs. Revue des Sciences de Gestion, 48(259/260), 10.

Chesbrough, H. (2010). Open Business Models. Boston, USA: Harvard Business School Press.

Chesbrough, H. (2011). Open Services Innovation. San Francisco, USA: Jossey Bass. A Wiley Imprint.

Davenport, T. y Prusak, L. (2001). Conocimiento en acción. Cómo las organizaciones manejan lo que saben (1. ${ }^{\mathrm{a}}$ ed.). Buenos Aires: Prentice Hall.

De la Fuente J.R. Entrevista sobre Educación. Gaceta UNAM, 9 (26 de septiembre de 2002).

Del Brío, J. Á., Fernández, E. y Junquera, B. (2005). Dificultad de Imitación de las Capacidades Medioambientales y Ventaja Competitiva. Un estudio empírico. Revista Europea de Dirección y Economía de Empresa, 14(4), 59-80.

Delgado, J. y Garibotti, H. (2005). Políticas de promoción de la industria del software. Universidad del CEMA.

Deng, X. y Chi, L. (2012). Understanding postadoptive behaviors in information systems use: A longitudinal analysis of system use problems in the business intelligence context. Journal of Management Information Systems, 29(3), $291-326$.

Duran M. Auditoria general d'una empresa d'alta tecnologia com a procediment inicial en la implementació d'una estratègia de formació continuada: la gestió del coneixement [tesis doctoral]. Barcelona, 2002.

Edvinsson, L., Hofman-Bang, P. y Jacobsen, K. (2005). Intellectual capital in waiting — a strategic IC challenge. Handbook of Business Strategy, 6(1), 133-140.

EFQM. European Foundation for Quality Management [consultado 1 Oct 2010]. Disponible en: http://www.efqm.org

Elbashir, M. Z., Collier, P. A. y Sutton, S. G. (2011). The role of organizational absorptive capacity in strategic use of business intelligence to support integrated management control systems. The Accounting Review, 86(1), $155-184$.

Esser, K., Hillebrand, W., Messner, D. y Meyer-Stamer, J. (1996). Competitividad internacional de las empresas y politicas requeridas: Competitividad sistémica. Berlin: Instituto Alemán de Desarrollo.

Fernández Nogales, Á. (1998). Investigación de mercados: obtención de información. Madrid: Ed. Civitas. Biblioteca Civitas Economía y Empresa. Colección Empresa.

Freire, P. (1973). La educación como práctica de la libertad. Buenos Aires, Argentina: Siglo XXI.

Gallego, D. y Ongallo, C. (2004). Conocimiento y gestión. Madrid: Pearson Education.

García, G. (2009). El concepto de competitividad sistémica. Revista Universidad de Sonora.

Goebbels, J. P. (1938). Der Rundfunk als achte Grossmacht — The radio as the eigth great power. Signale der neuen Zeit. 25 ausgewählte Reden von Dr. Joseph Goebbels, 197-207.

Goitia, S., Sáenz-de-Lacuesta, S. y Bilbao, M. (2008). Implantación de sistemas de información empresarial. El Profesional de la Información, 17(5), 540-545.

Grant, R. (1996). Toward a knowledge-base theory of the firm. Strategy Management Journal, 17, 109-122.

Hansen, M. T., Nohria, N. y Tierney, T. (1999). What's your strategy for managing knowledge? Harvard Business Review, 77(2), 106-116.

Hernández Sampieri, R., Fernández Collado, C. y Baptista Lucio, P. (2014). Metodología de la investigación (6. ${ }^{\mathrm{a}}$ ed.). México: McGraw Hill/Interamericana.

Kane, H., Ragsdell, G. y Oppenheim, C. (2006). Knowledge Management Methodologies. The Electronic Journal of Knowledge Management, 4(2), 141-152.

Kaplan, R. S. y Norton, D. P. (2006). Balanced Scorecard. USA: Thomson.

Kogut, B. y Zander, U. (1992). Knowkedge of the firm, combinative capabilities, and the replication of technology. Organization Science, 3(3), 383-397. 
Krueger, R.A. \& Casey, M.A. (2000). Focus Groups. A Practical Guide for Applied Research (3ra. Edición). Thousand Oaks, CA: Sage Publications, 206 p, ISBN 0-7619-2070-6.

Kumar, N. y Puranam, P. (2012). Injecting intelligence. Business Strategy Review, 23(3), 48-54.

Larson, B. (2009). Delivering Business Intelligence. New York: McGraw Hill.

Marsal, M. y Molina, J. (2002). La gestión del conocimiento en las organizaciones. Madrid: Colección de Negocios, Empresa y Economía. Libros en red.

Medellín Cabrera, E. A. (2010). Gestión tecnológica en empresas innovadoras mexicanas [U. d. Paulo, editor]. RAI Revista de Administração e Inovação, 7(3), 58-78.

Meyer-Stamer J. (2008). Systemic Competitiveness and Local Economic Development. Duisburg: Shamim Boadhanya (ed.), Large Scale Systemic Change: Theories, Modeling and Practices.

Michel, G. (2006). Existencia y método. México, D.F.: Castellanos Editores.

Michelo, J., Medellin, E., Hidalgo, A. y Jassó, J. (2008). Conocimiento e innovación. México: UAM-UNAM-Plaza y Valdés.

Mircea, M. A. (2009). Intelligence. Using business rules in business. Journal of Applied Quantitative Methods, 4(3), 12.

Moss, L. T. y Atre, S. (2003). Business Intelligence Roadmap: The Complete Project Lifecycle for Decision-Support Applications. USA: Addison Wesley.

Navarro, M. Á. y Bonilla, D. N. (2003). Gestión del conocimiento y servicios de inteligencia: la dimensión estratégica de la información. El Profesional de la Información, 12(4), 13.

Nemutanzhela, P. y Iyamu, T. (2011). A framework for enhancing the information system innovation: Using competitive intelligence. Electronic Journal of Information Systems Evaluation, 14(2), 242-253.

Nonaka, I. y Takeuchi, H. (1999). The knowledge creating company: How japanese companies create the dynamics of innovation. New York: Oxford University.

Nunnally J C. (1967) Psychometric theory. New York: McGraw Hill, 640 p.[University of Chicago, Chicago, IL].

Pavez Salazar, A. A. (2000). La gestión del conocimiento en las organizaciones. Documento en línea. Disponible en: http://www.gestiondelconocimiento.com/documentos2/apavez/gdc.htm. Consultado el día 13 de octubre de 2013.

PRODUCEN. (2008). Reporte de Investigación del Clúster de Tecnologías de Información. Tijuana: Autor.

Railean, L. (2011). Ensuring Competitive Advantage in SMEs in the construction industry in Romania through Technological Innovation. Young Economists Journal/Revista Tinerilor Economisti, 9(17), 110-117.

Rodríguez Gómez, D. (2006). Modelos para la creación y gestión del conocimiento: Una aproximación teórica. Educar, 37, 25-39.

Saint-Onge, H. (1996). Tacit knowledge: The key to the strategic aligment of intellectual capital. Strategy and Leadership, 24(2).

Sallis, E. y Jones, G. (2002). Knowledge Management in Education: Enchanging Learning and Education. Londres: Kogan Page Limited.

Shapiro, C. y Varian, H. (1999). El dominio de la información: una guía estratégica para la economía de la red. Barcelona: Antoni Bosch Editor.

Surma, J. (2011). Business Intelligence: Making Decisions through Data Analytics. Boston, USA: Business Expert Press, LLC.

Tiwana, A. (2002). The knowledge management toolkit: Ochestrating IT, strategy, and knowledges platforms. Upper Sadder River, N.J: Prentice Hall.

UNESCO. (2005). In M. Jouve (Ed.), Hacia las Sociedades del Conocimiento. París, Francia: Organización de las Naciones Unidas para la Educación, la Ciencia y la Cultura.

Villarreal, R. (2006). Competitividad en la Era del Conocimiento. México, D.F., México: Centro de Capital Intelectual y Competitividad.

Zapata-Cantú, L. E. (2004). Las determinantes de la generación y la transferencia del conocimiento en pequeñas y medianas empresas del sector de tecnologías de información en Barcelona [tesis doctoral]. Barcelona: Universitat Autónoma de Barcelona.

Zittoun, T. (2008). Learning through transitions: The role of institutions. Europan Journal of Psychology of Education, $165-181$. 